\title{
Space-Time Invariant Measures, Entropy, and Dimension for Stochastic Ginzburg-Landau Equations
}

\author{
Jacques Rougemont \\ Department of Mathematics, Heriot-Watt University, Edinburgh EH14 4AS, United Kingdom. \\ J.Rougemont@ma.hw.ac.uk
}

\begin{abstract}
We consider a randomly forced Ginzburg-Landau equation on an unbounded domain. The forcing is smooth and homogeneous in space and white noise in time. We prove existence and smoothness of solutions, existence of an invariant measure for the corresponding Markov process and we define the spatial densities of topological entropy, of measure-theoretic entropy, and of upper box-counting dimension. We prove inequalities relating these different quantities. The proof of existence of an invariant measure uses the compact embedding of some space of uniformly smooth functions into the space of locally square-integrable functions and a priori bounds on the semi-flow in these spaces. The bounds on the entropy follow from spatially localised estimates on the rate of divergence of nearby orbits and on the smoothing effect of the evolution.
\end{abstract}




\section{Introduction}

The use of dynamical system techniques and ideas in the study of extended partial differential equations has proved extremely fruitful in the past, see for example P. Collet's talk at ICM'98, [C2] (where he also emphasises the limitations of such an approach). However, until now only results using topological or geometric properties of the dynamics have been used (like invariant manifolds, bifurcation theory, topological entropy, Hausdorff dimension). That is to say, extended dynamical systems are usually regarded as topological dynamical systems. In contrast, most of the very deep results in finite dimensional dynamical systems use measure-theoretic ideas, namely ergodic theory (as advocated for instance in the review by L.-S. Young at the ICMP in 1997, [Y2]).

One of the favourite models of infinite dimensional dynamical systems studied recently is the Ginzburg-Landau equation. It appears as a generic normal form describing the amplitude of periodic bifurcated solutions (see [C2]) and it is also believed to be a good example of spatiotemporally chaotic dynamics [LO]. It is known that its attractor is infinite-dimensional and has positive $\varepsilon$-entropy (see [CE1, CE2, CE3, Ro]).

Here we propose to use random perturbations to obtain, by probabilistic techniques, the existence of invariant measures for the corresponding random dynamical system. The existence result is based on the observation by J. Ginibre and G. Velo that the Ginzburg-Landau equation has global solutions both in uniformly local Sobolev spaces and in local $\mathrm{L}^{2}$ space. Their proofs go through to the stochastic case without much effort, if we assume the noise to be smooth in space. Since uniformly local Sobolev spaces of sufficiently high order are compactly embedded into local $\mathrm{L}^{2}$ space, we get the Feller property of the semi-group and the tightness of the Cesàro means therefore existence of an invariant measure (by standard arguments for stochastic differential equations, see [DZ1]). These measures are also translation invariant, because the noise, the deterministic part of the equation and the spaces used are all translation invariant. We refer to the property of being invariant under the time evolution as "stationarity" and the invariance under space translations as "homogeneity" of the measure, following Vishik and Fursikov [VF].

In a second part of the paper we define the topological entropy and the measure-theoretic entropy, or rather their spatial densities, since both quantities are extensive (this has been discovered in this context by Collet and Eckmann in [CE1], see e.g. [Ru] for earlier similar ideas). Usual inequalities from ergodic theory can be proved in this case and the Collet-Eckmann bound on the topological entropy is also valid (see [CE2]).

The paper is organised as follows: In Section 2, we set the model and the functional analysis background needed for the remainder of the paper. The main results of the paper are summarised in Section 3. In Section 4 we obtain uniform bounds on the solutions in Sobolev spaces, these bounds being then used in Section 5 to prove the existence of invariant measures. Section 6 is devoted to the results on existence and properties of the (measure-theoretic and topological) entropies. Various technical proofs have been relegated to Sections $7-12$.

We finish this introduction by commenting on the fact that many new results on invariant measures for nonlinear PDEs have recently appeared. We mention for instance [BKD, FM], EH], $\mathrm{KS}$, Ku, Ma, S]. To the best of our knowledge the present work is the first where the model considered enjoys: infinite volume (hence continuous spectrum without gap), genuinely nonlin- 
ear interaction, homogeneous noise (hence infinite supply of energy at each time), non-trivial deterministic dynamics (e.g. the attractor of the deterministic Ginzburg-Landau is infinite dimensional). However, we are still unable to prove uniqueness of the invariant measure (i.e. an ergodicity result, as for example in [KS, E1, FM, Ma, BKD, DZ2]).

Acknowledgements. This work was supported by the Fonds National Suisse. I am grateful to Sergei Kuksin, Armen Shirikyan, and Martin Hairer for their comments and suggestions.

\section{Model and Definitions}

We consider equations of the form

$$
\begin{aligned}
& \mathrm{d} u=\left((1+\mathrm{i} \alpha) \Delta u+u-(1+\mathrm{i} \beta)|u|^{2 q} u\right) \mathrm{d} t+\xi \mathrm{d} w(t), \\
& u(x, t) \in \mathbf{C}, \quad x \in \mathbf{R}^{d}, \quad t \geq 0, \quad \alpha, \beta \in \mathbf{R},
\end{aligned}
$$

where $w(t)$ is a Wiener process and Eq.(2.1) is understood as an Itô stochastic differential in $t$. For a while we simply assume $\xi(\cdot, t) \in \mathcal{C}_{\mathrm{b}}^{\infty}\left(\mathbf{R}^{d}\right)$ uniformly in $t$ and it is adapted to the Wiener process in $t$. A specific example will be considered in Section 5. We also assume $u(\cdot, 0)=u_{0} \in$ $\mathcal{C}_{\mathrm{b}}^{\infty}\left(\mathbf{R}^{d}\right)$. We make the following

Hypothesis 2.1. We assume $d \leq 2, q>\frac{1}{2}$, and

$$
-(1+\alpha \beta)<|\alpha-\beta| \frac{\sqrt{2 q+1}}{q}, \quad|\beta| \leq \frac{\sqrt{2 q+1}}{q} .
$$

Remark. The second inequality in (2.2) implies the first one. We wrote the first one because it appears in this form in the proof of Proposition 4.4, while the second condition appears in the proof of Lemma [10.3, see Ginibre and Velo [GV1, GV2] for the most general results available in this direction.

We next introduce the function spaces used in this paper. Let

$$
\varphi_{\delta, y}(x)=\exp \left(-\sqrt{1+\delta^{2}|x-y|^{2}}\right) .
$$

The main feature of this function is that it belongs to $\mathrm{L}^{p}$ for all $p$ and

$$
\left\|\frac{\nabla^{n} \varphi_{\delta, y}}{\varphi_{\delta, y}}\right\|_{\infty}=A_{n} \delta^{n}<\infty
$$

for all $y \in \mathbf{R}^{d}$ and $n \in \mathbf{N}$. This function is used as weight on Sobolev and Lebesgue spaces:

Definition 2.2. The local Lebesgue space $\mathrm{L}_{\delta, y}^{2}$ is defined as the completion of $\mathcal{C}_{\mathrm{b}}^{\infty}$ (bounded smooth functions) in the norm induced by the scalar product

$$
(f, g)_{\delta, y}=\int \varphi_{\delta, y}(x) \overline{f(x)} g(x) \mathrm{d} x .
$$


The local Sobolev spaces $\mathrm{H}_{\delta, y}^{m}$ are defined as

$$
\mathrm{H}_{\delta, y}^{m}=\left\{f: \nabla^{k} f \in \mathrm{L}_{\delta, y}^{2}, k=0, \ldots, m\right\} .
$$

The uniformly local Sobolev spaces $\mathrm{H}_{\mathrm{ul}}^{m}$ are defined as the completion of $\mathcal{C}_{\mathrm{b}}^{\infty}$ in the norm

$$
\|f\|_{\mathrm{H}_{\mathrm{ul}}^{m}}^{2}=\sum_{k=0}^{m} \sup _{y \in \mathbf{R}^{d}}\left(\nabla^{k} f, \nabla^{k} f\right)_{\delta, y} .
$$

Remark that $\mathrm{H}_{\mathrm{ul}}^{m}$ is actually independent of $\delta>0$, since the following inclusion holds:

$$
\mathrm{H}_{\mathrm{ul}}^{m}=\bigcap_{y \in \mathbf{R}^{d}} \mathrm{H}_{\delta, y}^{m} \subset \bigcap_{\delta>0} \mathrm{H}_{\delta, y}^{m} .
$$

Usual Sobolev embeddings hold [Ad], for example if $m>d / 2$, the inequality

$$
\|f\|_{\infty}^{2} \leq C \delta\|f\|_{\mathrm{H}_{\mathrm{ul}}^{m}}^{2}
$$

implies the continuous embedding $\mathrm{H}_{\mathrm{ul}}^{m} \hookrightarrow \mathrm{L}^{\infty}$. Moreover, by the Rellich-Kondrachov Theorem [Ad],

$$
\mathrm{H}_{\delta, y}^{m+k} \hookrightarrow \mathrm{H}_{\delta^{\prime}, y}^{m}
$$

is compact if $k>d / 2$ and $0<\delta<\delta^{\prime}$ (see Section [11).

Notations. Throughout the paper, $\bar{z}$ denotes the complex conjugate of $z, f_{t}(x) \equiv f(x, t)$ hence $\left\|f_{t}\right\|_{\mathcal{X}}$ is the norm of $f(x, t)$ in the space $\mathcal{X}(\mathrm{d} x)\left(e . g . \mathcal{X}=\mathrm{L}^{2}\right)$. Norms in Lebesgue spaces $\mathrm{L}^{p}$ are denoted $\|\cdot\|_{p}\left(\|\cdot\|\right.$ is usually the norm on $\mathrm{L}_{\delta, y}^{2}$ for the current choice of $\delta$ and $\left.y\right)$. Expectations and probabilities with respect to the Wiener measure are denoted $\mathbf{E}$ and $\mathbf{P}$. We denote the integer part of the positive real $x$ by $[x] \equiv \max \{n \in \mathbf{N}: n \leq x\}$. Symbols $C, C_{1}, C_{2}, \ldots, c, c_{1}, c_{2}, \ldots$ usually denote generic numerical constant. The product $f \star g$ means the convolution of the functions $f$ and $g$. The cube of side $L$ and centre 0 in $\mathbf{R}^{d}$ is $Q_{L}=\left[-\frac{1}{2} L, \frac{1}{2} L\right]^{d}$.

\section{Summary of Results}

In this section, we describe in a rather informal way the main results of this paper. The first result (Section (4) is the following theorem of existence of smooth bounded solutions to Eq.(2.1): Theorem A. If Hypothesis 2.1 holds, then Eq.(2.1) with initial data $u_{0} \in \mathcal{C}_{\mathrm{b}}^{\infty}$ has a unique solution $u(x, t)=u_{t}(x)$. For all real $p \geq 1$ and integer $m$, there is a $B_{p, m}<\infty$ such that for all $t>0$ :

$$
\mathbf{E}\left\|u_{t}\right\|_{\mathrm{H}_{\mathrm{ul}}^{m}}^{p} \leq B_{p, m} .
$$

The proof relies on well-known estimates [GV1, Mi, C1] using the dissipative nature of the nonlinear term in Eq.(2.1) for the deterministic part and on Itô's Lemma to treat the stochastic 
term. Actually, in the evolution equation for $\mathbf{E}\left\|u_{t}\right\|_{\mathrm{H}_{\mathrm{ul}}^{m}}^{p}$, Itô's Lemma only generates terms which are dominated by the nonlinear dissipative term and this implies that the techniques which were developed for the deterministic equation are applicable.

By Lemma 10.3 we can extend the existence result to the space $\mathrm{L}_{\delta, y}^{2}$, hence we can define the Markovian Feller semi-group $\mathcal{P}_{t}$ acting on $\mathcal{C}_{\mathrm{b}}\left(\mathrm{L}_{\delta, y}^{2}, \mathrm{C}\right)$ for any specific choice of $y$, for example $y=0$ :

$$
\left(\mathcal{P}_{t} f\right)(u)=\int_{\mathrm{L}_{\delta, 0}^{2}} f(\eta) \mathbf{P}\left(u_{t} \in \mathrm{d} \eta\right) .
$$

An invariant measure for Eq.(2.1) is a fixed point of the dual semi-group $\mathcal{P}_{t}^{*}$. We next assume that $\xi$ is an homogeneous process adapted to the Brownian motion. Since $\mathrm{H}_{\mathrm{ul}}^{m}$ is compactly embedded into $\mathrm{L}_{\delta, y}^{2}$ for $m>d / 2$ (see (2.7)) the following theorem (see Section 5) is an immediate consequence of Theorem A by the Prokhorov and Krylov-Bogolyubov Theorems (see [Ar], $\mathrm{VF}$, DZ2]):

Theorem B. There exists at least one invariant measure $\mu$ for Eq.(2.1). This measure is homogeneous in $x$ and its support is contained in $\bigcap_{m \geq 0} \mathrm{H}_{\mathrm{ul}}^{m}$.

Finally, in Section 6, we define the random attractor (see [CDF])

$$
\begin{aligned}
\mathcal{A}_{\omega} & =\bigcup_{R>0} \mathcal{A}(\omega, R)^{\mathrm{H}_{\mathrm{ul}}^{m}}, \\
\mathcal{A}(\omega, R) & =\bigcap_{T>0} \bigcup_{t>T} \Phi_{\theta^{-t} \omega}^{t}\left(B_{R}\right)^{\mathrm{H}_{\mathrm{ul}}^{m}} .
\end{aligned}
$$

Here and below $\Phi_{\omega}^{t}$ is the semi-group generated by Eq.(2.1), $\theta^{t}$ is the time-shift of the noise, and $T_{x}$ the group of spatial translations. Moreover $B_{R} \subset \mathrm{H}_{\mathrm{ul}}^{m}$ is the ball of radius $R$ and centre 0 . We introduce the following dynamical observables (see [KH], [LQ]):

$$
\begin{aligned}
h_{\mathrm{top}} & =\lim _{\varepsilon \rightarrow 0} \lim _{L \rightarrow \infty} \frac{1}{L^{d}} \lim _{n \rightarrow \infty} \frac{1}{n \tau} \int \log N_{\omega, n, \tau, Q_{L}, \varepsilon} \mathbf{P}(\mathrm{d} \omega), \\
h_{\mu} & =\lim _{\varepsilon \rightarrow 0} \lim _{L \rightarrow \infty} \frac{1}{L^{d}} \lim _{n \rightarrow \infty} \frac{1}{n \tau} \int H_{\mu}\left(\bigvee_{x \in \mathbf{Z}^{d} \cap Q_{L}} \bigvee_{k=0}^{n-1} \Phi_{\omega}^{-k \tau} T_{-x}\left(\Sigma_{\theta^{k \tau} T_{x} \omega, \varepsilon}\right)\right) \mathbf{P}(\mathrm{d} \omega), \\
\mathcal{H}_{\varepsilon} & =\lim _{L \rightarrow \infty} \int \frac{\log M_{\varepsilon, Q_{L}, \omega}}{L^{d}} \mathbf{P}(\mathrm{d} \omega), \\
d_{\text {up }} & =\limsup _{\varepsilon \rightarrow 0} \frac{\mathcal{H}_{\varepsilon}}{\log \varepsilon^{-1}},
\end{aligned}
$$

where $N_{\omega, n, \tau, Q, \varepsilon}$ is the cardinality of a minimal $(n, \varepsilon)$-cover of $\left.\mathcal{A}_{\omega}\right|_{Q}, \Sigma_{\omega, \varepsilon}$ is a sequence of partitions of $\mathcal{A}_{\omega}$ in sets of diameter at most $\varepsilon$ in the metric of $\mathrm{L}^{\infty}\left(Q_{1}\right), M_{\varepsilon, Q, \omega}$ is the least cardinality of an $\varepsilon$-cover of $\left.\mathcal{A}_{\omega}\right|_{Q}$ and $Q_{L}=\left[-\frac{1}{2} L, \frac{1}{2} L\right]^{d}$ (see Section 6 for detailed definitions).

The quantities in Eq.(3.1) are called respectively the topological entropy, the metric or measure-theoretic entropy [ $\mathrm{KH}]$, the Kolmogorov-Tikhomirov $\varepsilon$-entropy [KT] and the upper (boxcounting) dimension. It is important to note that the above numbers are all spatial densities (limit 
as $L \rightarrow \infty$ of quantities divided by $L^{d}$ ) although the limits are not taken in the most natural order. They are thus spatially localised versions of the usual entropies and dimensions.

We then prove the following estimates:

Theorem C. There is a $\gamma<\infty$ such that $h_{\mu} \leq h_{\mathrm{top}} \leq \gamma d_{\mathrm{up}}<\infty$.

The proof that all the various limits in Eq.(3.1) exist relies on standard subadditive bounds [KH]. The upper bound on $d_{\text {up }}$ follows from spatially localised estimates of the rate of divergence of nearby orbits (Lemma 6.7) as well as the smoothing action of the evolution (see Section 7, in particular Lemma 7.1]). It is similar to the proof of the deterministic case [CE2].

\section{Bounded Smooth Solutions}

Our first result in this paper is the existence (and uniqueness by Lemma 10.1) of smooth bounded solutions to Eq.(2.1).

Theorem 4.1. If Hypothesis 2.1 holds, then Eq.(2.1) with initial data $u_{0} \in \mathcal{C}_{\mathrm{b}}^{\infty}$ has a unique solution $u(x, t)=u_{t}(x)$. For all real $p \geq 1$ and integer $m$, there is a $B_{p, m}<\infty$ such that for all $t>0$ :

$$
\mathbf{E}\left\|u_{t}\right\|_{\mathrm{H}_{\mathrm{ul}}^{m}}^{p} \leq B_{p, m}
$$

Remark. This proof is amply simplified by our assumptions on the regularity of $\xi_{t}$ in Eq.(2.1). A much more general theory of stochastic PDEs on $\mathbf{R}^{d}$ can be found, for example, in Krylov [ $\left.\mathrm{Kr}\right]$. Funaki [F1, F2] has studied a similar equations with stronger assumptions on the nonlinearity and Eckmann-Hairer [EH] have recently proved a similar result for stochastic forcings with finite energy.

Proof. In the first part of the proof, we fix $y \in \mathbf{R}^{d}$ and $\delta>0$ such that $A_{1} \delta+A_{2} \delta^{2}<1$ (see Eq.(2.4)). We write $\|\cdot\|$ and $(\cdot, \cdot)$ for the norm and scalar product in the corresponding space $\mathrm{L}_{\delta, y}^{2}$. All bounds will actually turn out to be uniform in $y$. We stress that scalar products denoted $(\cdot, \cdot)$ contain the weight $\varphi_{\delta, y}$ (see Definition 2.2) hence integration by parts produces commutators of the form $\nabla \varphi_{\delta, y} / \varphi_{\delta, y}$. From now on, we also write $\varphi$ for $\varphi_{\delta, y}$.

Let $\mathcal{L}=(1+\mathrm{i} \alpha) \Delta+1$. For $f \in D_{m}(\Delta) \subset \mathrm{H}_{\delta, y}^{m}$ (the domain of the closure in $\mathrm{H}_{\delta, y}^{m}$ of $\Delta$ with core $\left.\mathcal{C}_{\mathrm{b}}^{\infty}\right)$, the following holds by Eq.(2.4):

$$
\begin{aligned}
& \operatorname{Re}\left(\nabla^{m} f, \nabla^{m} \mathcal{L} f\right) \\
& =-\left(\nabla^{m+1} f, \nabla^{m+1} f\right)+\left(\nabla^{m} f, \nabla^{m} f\right)+\operatorname{Re}\left(\nabla \varphi \varphi^{-1} \nabla^{m} f,(1+\mathrm{i} \alpha) \nabla^{m+1} f\right) \\
& \leq-\frac{1}{2}\left(\nabla^{m+1} f, \nabla^{m+1} f\right)+\left(1+\frac{1}{2}\left(1+\alpha^{2}\right)\right)\left(\nabla^{m} f, \nabla^{m} f\right) .
\end{aligned}
$$

Namely $\mathcal{L}-\left(1+\left(1+\alpha^{2}\right) / 2\right)$ is a dissipative operator hence by the Lumer-Phillips Theorem [Y1], $\mathcal{L}$ generates a strongly continuous quasi-bounded semi-group $\exp (t \mathcal{L})$ on $\mathrm{H}_{\delta, y}^{m}$, with

$$
\left\|e^{t \mathcal{L}}\right\|_{\mathrm{H}_{\delta, y}^{m} \rightarrow \mathrm{H}_{\delta, y}^{m}} \leq e^{c t}
$$


for some $c<\infty$. Remark that we may have chosen $\delta$ such that $c=1+\varepsilon$ for arbitrarily small $\varepsilon>0$.

We define mild solutions to Eq.(2.1) in $\mathrm{H}_{\delta, y}^{m}$ by the Duhamel formula (and the Itô integral):

$$
z_{t}=e^{t \mathcal{L}} z_{0}-(1+\mathrm{i} \beta) \int_{0}^{t} e^{(t-s) \mathcal{L}}\left|z_{s}\right|^{2 q} z_{s} \mathrm{~d} s+\int_{0}^{t} e^{(t-s)} \mathcal{L}_{\xi_{s}} \mathrm{~d} w_{s}
$$

We let $P_{M}: \mathbf{R}^{+} \rightarrow \mathbf{R}^{+}$be a smooth cutoff function satisfying $P_{M}(x)=1$ if $x<M$ and $P_{M}(x)=0$ if $x>M+1$. We introduce this cutoff into the nonlinear term above, effectively rendering the nonlinearity uniformly Lipschitz:

$$
\widetilde{z}_{t}=e^{t \mathcal{L}} \widetilde{z}_{0}-(1+\mathrm{i} \beta) \int_{0}^{t} e^{(t-s) \mathcal{L}} P_{M}\left(\left|\widetilde{z}_{s}\right|\right)\left|\widetilde{z}_{s}\right|^{2 q} \widetilde{z}_{s} \mathrm{~d} s+\int_{0}^{t} e^{(t-s) \mathcal{L}} \xi_{s} \mathrm{~d} w_{s} .
$$

We next define the random stopping time $\tau(R)$ by

$$
\tau(R)=\min \left\{t>0:\left\|\widetilde{z}_{t}\right\|_{\infty} \geq R\right\} .
$$

We fix arbitrarily a positive number $R<M$, and if $\chi_{I}$ denotes the characteristic function of the set $I$, we consider the following integral equation for $t<\tau(R)$ :

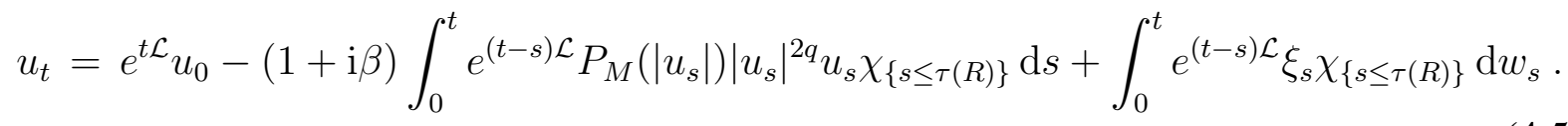

The following is a simple consequence of our construction:

Lemma 4.2. There is almost surely a unique function $u_{t}$ satisfying Eq.(4.5), this function is independent of $M>R$ and it also satisfies Eq.(4.2) for $t<\tau(R)$.

Proof. See [DZ1, $\mathrm{Ku}$ ] for the properties of the stochastic convolution and Section 10 for the contraction argument needed to prove uniqueness.

The remaining part of the proof of Theorem 4.1 follows very closely the paper by Mielke [Mi] which is based on [BGO, C1, GV1, GV2]. We first establish uniform bounds in $\mathrm{L}_{\delta, y}^{2}$.

Lemma 4.3. For all $\delta>0$ and $p \geq 1$, there are $C_{0, p}(\delta)$ such that the following bound holds for all $t>0$ and all $y \in \mathbf{R}^{d}$ :

$$
\mathbf{E}\left\|u_{t}\right\|_{\mathrm{L}_{\delta, y}^{2}}^{p} \leq C_{0, p}(\delta)
$$

Proof. We first estimate the square of the norm in $\mathrm{L}_{\delta, y}^{2}=\mathrm{L}^{2}$. By Itô's formula, we have

$$
\begin{aligned}
\mathrm{d}\left\|u_{t}\right\|^{2}= & -2\left\|\nabla u_{t}\right\|^{2} \mathrm{~d} t-2 \operatorname{Re}\left(\nabla \varphi \varphi^{-1} u_{t},(1+\mathrm{i} \alpha) \nabla u_{t}\right) \mathrm{d} t+2\left\|u_{t}\right\|^{2} \mathrm{~d} t \\
& -2 \operatorname{Re}\left(u_{t},(1+\mathrm{i} \beta)\left|u_{t}\right|^{2 q} u_{t}\right) \mathrm{d} t+\left\|\xi_{t}\right\|^{2} \mathrm{~d} t+2 \operatorname{Re}\left(u_{t}, \xi_{t}\right) \mathrm{d} w_{t} \\
\leq & -\left\|\nabla u_{t}\right\|^{2} \mathrm{~d} t+\left(2+\left(1+\alpha^{2}\right)\right)\left\|u_{t}\right\|^{2} \mathrm{~d} t-2\left(u_{t},\left|u_{t}\right|^{2 q} u_{t}\right) \mathrm{d} t \\
& +\left\|\xi_{t}\right\|^{2} \mathrm{~d} t+2 \operatorname{Re}\left(u_{t}, \xi_{t}\right) \mathrm{d} w_{t} \\
\leq & -\left\|\nabla u_{t}\right\|^{2} \mathrm{~d} t+C\left(\alpha,\left\|\xi_{t}\right\|, q\right) \mathrm{d} t-\left\|u_{t}\right\|^{2} \mathrm{~d} t+2 \operatorname{Re}\left(u_{t}, \xi_{t}\right) \mathrm{d} w_{t} .
\end{aligned}
$$


We integrate this last inequality over $t$ and take expectations. By standard arguments the expectation of the Itô integral vanishes (recall that we consider stopped solutions, Eq.(4.5), see [DZ1]) and we obtain

$$
\mathbf{E}\left\|u_{T}\right\|^{2} \leq \mathbf{E}\left\|u_{0}\right\|^{2}-\mathbf{E} \int_{0}^{T}\left\|\nabla u_{t}\right\|^{2} \mathrm{~d} t-\mathbf{E} \int_{0}^{T}\left(\left\|u_{t}\right\|^{2}-C\right) \mathrm{d} t .
$$

By Gronwall's inequality, this is

$$
\mathbf{E}\left\|u_{T}\right\|^{2} \leq \max \left\{C_{0,2},\left(\mathbf{E}\left\|u_{0}\right\|^{2}-C_{0,2}\right) e^{-T}+C_{0,2}\right\} .
$$

For higher powers of the $\mathrm{L}^{2}$ norm, we use Itô's formula again:

$$
\frac{1}{p} \mathrm{~d}\left\|u_{t}\right\|_{2}^{2 p}=\left\|u_{t}\right\|^{2 p-2} \mathrm{~d}\left\|u_{t}\right\|^{2}+2(p-1)\left\|u_{t}\right\|^{2 p-4}\left(\operatorname{Re}\left(u_{t}, \xi_{t}\right)\right)^{2} \mathrm{~d} t
$$

hence (after substituting the estimate (4.7))

$$
\mathbf{E}\left\|u_{T}\right\|_{2}^{2 p} \leq \mathbf{E}\left\|u_{0}\right\|_{2}^{2 p}-\mathbf{E} \int_{0}^{T}\left(\left\|u_{t}\right\|^{2 p}-C_{0,2 p}\right) \mathrm{d} t,
$$

which by Gronwall's inequality gives a uniform bound on $\left\|u_{t}\right\|^{p}$ for $p>2$. For $p \in[1,2)$, we use Jensen's inequality:

$$
\mathbf{E}\left\|u_{t}\right\|^{p} \leq\left(\mathbf{E}\left\|u_{t}\right\|^{2}\right)^{p / 2} \leq C_{0,2}^{p / 2}=C_{0, p} .
$$

If $u_{0}$ is uniformly bounded and because $\left\|\xi_{t}\right\|_{\mathrm{L}_{\delta, y}^{2}}^{p}$ is bounded uniformly in $y$ and $t$, we obtain the uniform bound in the spaces $\mathrm{L}_{\delta, y}^{2}$ for all $y$

$$
\sup _{t>0} \sup _{y \in \mathbf{R}^{d}} \mathbf{E}\left\|u_{t}\right\|_{\mathrm{L}_{\delta, y}^{2}}^{p} \leq C_{0, p}(\delta)
$$

which proves Lemma 4.3 .

Proposition 4.4. For all $\delta>0$ and $p \geq 1$, there are $C_{1, p}(\delta)$ such that the following bound holds for all $t>0$ and all $y \in \mathbf{R}^{d}$ :

$$
\mathbf{E}\left\|u_{t}\right\|_{\mathrm{H}_{\delta, y}^{1}}^{p} \leq C_{1, p}(\delta)
$$

Proof. We first consider the differential

$$
\begin{aligned}
\mathrm{d}\left\|\nabla u_{t}\right\|^{2}= & -2\left\|\Delta u_{t}\right\|^{2} \mathrm{~d} t-2 \operatorname{Re}\left(\nabla \varphi \varphi^{-1} \nabla u_{t},(1+\mathrm{i} \alpha) \Delta u_{t}\right) \mathrm{d} t+2\left\|\nabla u_{t}\right\|^{2} \mathrm{~d} t \\
& +2 \operatorname{Re}\left(\Delta u_{t},(1+\mathrm{i} \beta)\left|u_{t}\right|^{2 q} u_{t}\right) \mathrm{d} t+2 \operatorname{Re}\left(\nabla \varphi \varphi^{-1} \nabla u_{t},(1+\mathrm{i} \beta)\left|u_{t}\right|^{2 q} u_{t}\right) \mathrm{d} t \\
& +\left\|\nabla \xi_{t}\right\|^{2} \mathrm{~d} t+2 \operatorname{Re}\left(\nabla u_{t}, \nabla \xi_{t}\right) \mathrm{d} w_{t} \\
\leq & -2\left\|\Delta u_{t}\right\|^{2} \mathrm{~d} t+2\left\|\nabla u_{t}\right\|^{2} \mathrm{~d} t+2 \operatorname{Re}\left(\Delta u_{t},(1+\mathrm{i} \beta)\left|u_{t}\right|^{2 q} u_{t}\right) \mathrm{d} t \\
& +2\left(\sqrt{1+\alpha^{2}}\left\|\Delta u_{t}\right\|+\sqrt{1+\beta^{2}}\left\|\left|u_{t}\right|^{2 q+1}\right\|\right)\left\|\nabla u_{t}\right\| \mathrm{d} t \\
& +\left\|\nabla \xi_{t}\right\|^{2} \mathrm{~d} t-2 \operatorname{Re}\left(u_{t}, \varphi^{-1} \nabla\left(\varphi \nabla \xi_{t}\right)\right) \mathrm{d} w_{t},
\end{aligned}
$$


and we also compute the following differential that will help us to cancel out some of the terms above:

$$
\begin{aligned}
\frac{1}{q+1} \mathrm{~d}\left\|\left|u_{t}\right|^{q+1}\right\|^{2}= & 2 \operatorname{Re}\left(\left|u_{t}\right|^{2 q} u,(1+\mathrm{i} \alpha) \Delta u_{t}\right) \mathrm{d} t+2\left\|\left|u_{t}\right|^{q+1}\right\|^{2} \mathrm{~d} t \\
& -2\left\|\left|u_{t}\right|^{2 q} u_{t}\right\|^{2} \mathrm{~d} t+2 q\left(\operatorname{Re}\left(\left|u_{t}\right|^{q-1} u_{t}, \xi_{t}\right)\right)^{2} \mathrm{~d} t+2 \operatorname{Re}\left(\left|u_{t}\right|^{2 q} u_{t}, \xi_{t}\right) \mathrm{d} w_{t} \\
\leq & 2 \operatorname{Re}\left(\left|u_{t}\right|^{2 q} u,(1+\mathrm{i} \alpha) \Delta u_{t}\right) \mathrm{d} t+2\left\|\left|u_{t}\right|^{q+1}\right\|^{2} \mathrm{~d} t+2 q\left\|\xi_{t}\right\|^{2}\left\|\left|u_{t}\right|^{q}\right\|^{2} \mathrm{~d} t \\
& -2\left\|\left|u_{t}\right|^{2 q} u_{t}\right\|^{2} \mathrm{~d} t+2 \operatorname{Re}\left(\left|u_{t}\right|^{2 q} u_{t}, \xi_{t}\right) \mathrm{d} w_{t} .
\end{aligned}
$$

We take a convex combination of Inequalities (4.10) and (4.9) (here $\lambda \in[0,1])$ :

$$
\begin{aligned}
& \frac{1}{2}\left(\lambda \mathrm{d}\left\|\nabla u_{t}\right\|^{2}+\frac{(1-\lambda)}{q+1} \mathrm{~d}\left\|\left|u_{t}\right|^{q+1}\right\|^{2}\right) \\
& \leq\left(\lambda\left\|\nabla u_{t}\right\|^{2}+(1-\lambda)\left\|\left|u_{t}\right|^{q+1}\right\|^{2}\right) \mathrm{d} t+\left((1-\lambda) q\left\|\xi_{t}\right\|^{2}\left\|\left|u_{t}\right|^{q}\right\|^{2}+\frac{\lambda}{2}\left\|\nabla \xi_{t}\right\|^{2}\right) \mathrm{d} t \\
& \quad+\lambda\left(\sqrt{1+\alpha^{2}}\left\|\Delta u_{t}\right\|+\sqrt{1+\beta^{2}}\left\|\left|u_{t}\right|^{2 q+1}\right\|\right)\left\|\nabla u_{t}\right\| \mathrm{d} t \\
& \quad+\mathcal{M} \mathrm{d} t+\operatorname{Re}\left(-\lambda\left(u_{t}, \varphi^{-1} \nabla\left(\varphi \nabla \xi_{t}\right)\right)+(1-\lambda) b\left(\left|u_{t}\right|^{2 q} u_{t}, \xi_{t}\right)\right) \mathrm{d} w_{t} .
\end{aligned}
$$

The term denoted by $\mathcal{M}$ is treated separately:

$$
\begin{aligned}
\mathcal{M}= & -\left(\lambda\left\|\Delta u_{t}\right\|^{2}+(1-\lambda)\left\|\left|u_{t}\right|^{2 q+1}\right\|^{2}\right)+\operatorname{Re}(1-\mathrm{i}(\lambda \beta-(1-\lambda) \alpha))\left(\left|u_{t}\right|^{2 q} u_{t}, \Delta u_{t}\right) \\
\leq & -\varepsilon\left(\lambda\left\|\Delta u_{t}\right\|^{2}+\frac{(1-\lambda)}{q+1}\left\|\left|u_{t}\right|^{2 q+1}\right\|^{2}\right)-2(1-\varepsilon) \sqrt{\lambda(1-\lambda)}\left|\left(\left|u_{t}\right|^{2 q} u_{t}, \Delta u_{t}\right)\right| \\
& +\operatorname{Re}(1-\mathrm{i}(\lambda \beta-(1-\lambda) \alpha))\left(\left|u_{t}\right|^{2 q} u_{t}, \Delta u_{t}\right) \\
\equiv & -\varepsilon\left(\lambda\left\|\Delta u_{t}\right\|^{2}+\frac{(1-\lambda)}{q+1}\left\|\left|u_{t}\right|^{2 q+1}\right\|^{2}\right)+\widetilde{\mathcal{M}}(\varepsilon) .
\end{aligned}
$$

Under Hypothesis 2.1, there is an $\varepsilon>0$ such that $\widetilde{\mathcal{M}}(\varepsilon)$ is negative (see e.g. [GV1, M1, BGO]). The proof goes as follows: we first remark that integration by parts leads to

$$
\left(\left|u_{t}\right|^{2 q} u_{t}, \Delta u_{t}\right)=-\left(\frac{\nabla \varphi}{\varphi}\left|u_{t}\right|^{2 q} u_{t}, \nabla u_{t}\right)-(q+1) \int \varphi\left|u_{t}\right|^{2 q}\left|\nabla u_{t}\right|^{2}\left(1+\frac{q}{1+q} \frac{\bar{u}_{t}^{2}}{\left|u_{t}\right|^{2}} \frac{\nabla u_{t}^{2}}{\left|\nabla u_{t}\right|^{2}}\right) .
$$

The last bracket above is of the form $1+z$. Its argument can be estimated as follows: $\mid \arg (1+$ $z)|\leq \arcsin | z \mid=\arcsin \frac{q}{1+q} \equiv \theta$. We plug this into $\widetilde{\mathcal{M}}$ :

$$
\begin{aligned}
\widetilde{\mathcal{M}}(\varepsilon) \leq & -(q+1)\left|\left(\left|u_{t}\right|^{2 q},\left|\nabla u_{t}\right|^{2}\right)\right|\{(2(1-\varepsilon) \sqrt{\lambda(1-\lambda)}+\cos \theta)-|\lambda \beta-(1-\lambda) \alpha| \sin \theta\} \\
& +C(\alpha, \beta, \lambda, \varepsilon)\left\|\left|u_{t}\right|^{2 q+1}\right\|\left\|\nabla u_{t}\right\|
\end{aligned}
$$


The curly bracket above can be made positive by suitably choosing $\lambda$ and $\varepsilon$, namely we take $\lambda=\cos ^{2} \eta$, we optimise for $\eta$ and we obtain the following condition, which is obviously fulfilled for small $\varepsilon>0$ under Hypothesis 2.1 (remark that $1 / \tan \theta=\sqrt{2 q+1} / q$ ):

$$
-(1+\alpha \beta)-|\beta-\alpha| / \tan \theta+\varepsilon(2-\varepsilon) / \sin ^{2} \theta \leq 0
$$

(see Ginibre and Velo [GV1] for this argument, Mielke [Mi] has a slightly different formulation). We thus obtain

$$
\begin{aligned}
& \frac{1}{2}\left(\lambda \mathrm{d}\left\|\nabla u_{t}\right\|^{2}+\frac{(1-\lambda)}{q+1} \mathrm{~d}\left\|\left|u_{t}\right|^{q+1}\right\|^{2}\right) \\
& \leq-\varepsilon\left(\lambda\left\|\Delta u_{t}\right\|^{2}+\frac{(1-\lambda)}{q+1}\left\|\left|u_{t}\right|^{2 q+1}\right\|^{2}\right) \mathrm{d} t \\
& \quad+\left(C_{1}\left\|\nabla u_{t}\right\|^{2}+C_{2}\left\|\left|u_{t}\right|^{q+1}\right\|^{2}\right) \mathrm{d} t+\left(C_{3}\left\|\xi_{t}\right\|^{2}\left\|\left|u_{t}\right|^{q}\right\|^{2}+C_{4}\left\|\nabla \xi_{t}\right\|^{2}\right) \mathrm{d} t \\
& \quad+\left(C_{5}\left\|\Delta u_{t}\right\|+C_{6}\left\|\left|u_{t}\right|^{2 q+1}\right\|+C_{7}\right)\left\|\nabla u_{t}\right\| \mathrm{d} t \\
& \quad+\operatorname{Re}\left(-\lambda\left(u_{t}, \varphi^{-1} \nabla\left(\varphi \nabla \xi_{t}\right)\right)+(1-\lambda)\left(\left|u_{t}\right|^{2 q} u_{t}, \xi_{t}\right)\right) \mathrm{d} w_{t} \\
& \leq C \mathrm{~d} t-\frac{\varepsilon}{2}\left(\lambda\left\|\nabla u_{t}\right\|^{2}+\frac{(1-\lambda)}{q+1}\left\|\left|u_{t}\right|^{q+1}\right\|^{2}\right) \mathrm{d} t \\
& \quad+\operatorname{Re}\left(-\lambda\left(u_{t}, \varphi^{-1} \nabla\left(\varphi \nabla \xi_{t}\right)\right)+(1-\lambda)\left(\left|u_{t}\right|^{2 q} u_{t}, \xi_{t}\right)\right) \mathrm{d} w_{t},
\end{aligned}
$$

thanks to the following obvious inequality:

$$
-\left\|\Delta u_{t}\right\|^{2} \leq-\rho\left\|\nabla u_{t}\right\|^{2}+C \rho^{2}\left\|u_{t}\right\|^{2}
$$

which holds for all $\rho>0$ and for some $C>0$. As before, we take expectations, integrate over $t$ and we use Gronwall's inequality to find out the following bound:

$$
\begin{aligned}
& \max \left\{\mathbf{E}\left\|\nabla u_{T}\right\|^{2}, \mathbf{E}\left\|\left|u_{T}\right|^{q+1}\right\|^{2}\right\} \\
& \leq \max \left\{C_{1,2},\left(\mathbf{E}\left\|\nabla u_{0}\right\|^{2}+\mathbf{E}\left\|\left|u_{0}\right|^{q+1}\right\|^{2}-C_{1,2}\right) e^{-\varepsilon T}+C_{1,2}\right\} .
\end{aligned}
$$

This and Lemma 4.3 prove Proposition 4.4 .

We next consider solutions $z(x, t)$ to Eq.(4.2) with bounded initial condition. Proposition 4.4 on $u(x, t)$ implies the following:

Proposition 4.5. For all $p \geq 1$, there is a $C_{\infty, p}$ such that for all $t>0$, the following holds

$$
\mathbf{E}\left\|z_{t}\right\|_{\infty}^{p} \leq C_{\infty, p}
$$

Proof. By the bound (2.6), if $d=1$ then Proposition 4.4 implies the bound (4.13) for stopped solutions. If $d=2$ we need a bound in $\mathrm{H}_{\delta, y}^{2}$. This is easily achieved with the help of a GagliardoNirenberg inequality which we prove in Section 12: 
Lemma 4.6. Let $f \in \mathrm{H}_{\mathrm{ul}}^{3}\left(\mathbf{R}^{2}\right)$. For all $K>0$ there are $C(K)$, $\eta$ such that

$$
\begin{aligned}
& \int \varphi_{\delta, y}(x)\left|\Delta\left(|f(x)|^{2 q} f(x)\right) \overline{\Delta f(x)}\right| \mathrm{d} x \\
& \leq \frac{1}{K} \int \varphi_{\delta, y}\left|\nabla^{3} f(x)\right|^{2} \mathrm{~d} x+C(K)\left(\sup _{y} \int \varphi_{\delta, y}(x)|f(x)|^{2(q+1)} \mathrm{d} x\right)^{\eta} .
\end{aligned}
$$

We use Inequality (4.11) (with $u_{t}$ replaced by $\nabla u_{t}$ ), Lemma 4.6, and the estimate (4.12) to bound the time derivative of $\left\|\Delta u_{t}\right\|_{\mathrm{L}_{\delta, x}^{2}}^{2}$ :

$$
\begin{aligned}
\frac{1}{2} \mathrm{~d}\left\|\Delta u_{t}\right\|_{\mathrm{L}_{\delta, x}^{2}}^{2} \leq & -\frac{1}{2}\left\|\nabla^{3} u_{t}\right\|_{\mathrm{L}_{\delta, x}^{2}}^{2} \mathrm{~d} t+\left(1+\left(1+\alpha^{2}\right)\right)\left\|\Delta u_{t}\right\|_{\mathrm{L}_{\delta, x}^{2}}^{2} \mathrm{~d} t \\
& +C\left(1+\beta^{2}\right) \sup _{y}\left\|\left|u_{t}\right|^{q+1}\right\|_{\mathrm{L}_{\delta, y}^{2}}^{2 \eta} \mathrm{d} t+\frac{1}{2}\left\|\Delta \xi_{t}\right\|_{\mathrm{L}_{\delta, x}^{2}}^{2} \mathrm{~d} t+\left(\Delta u_{t}, \Delta \xi_{t}\right)_{\delta, x} \mathrm{~d} w_{t} \\
\leq & \left(-\frac{1}{2} \mathrm{~d}\left\|\Delta u_{t}\right\|_{\mathrm{L}_{\delta, x}^{2}}^{2}+C\right) \mathrm{d} t+\left(u_{t}, \varphi^{-1} \Delta\left(\varphi \Delta \xi_{t}\right)\right)_{\delta, x} \mathrm{~d} w_{t},
\end{aligned}
$$

where $C$ depends on the parameters in Eq.(2.1) (including $\xi_{t}$ ) and on $\left\|u_{t}\right\|_{\mathrm{L}_{\delta, x}^{2}}^{p},\left\|\left|u_{t}\right|^{q+1}\right\|_{\mathrm{L}_{\delta, x}^{2}}^{\eta}$, and $\left\|\nabla u_{t}\right\|_{\mathrm{L}_{\delta, x}^{2}}^{2}$ which satisfy Bounds (4.6) and (4.12) (or rather some extension of it to deal with the power $\eta$ ). By the usual Gronwall inequality, this proves (4.13) for stopped solutions (Eq.(4.5)).

We now choose a very large $n_{0} \gg C_{0,2}+C_{1,2}+C_{2,2}$ and we let

$$
\mathcal{E}_{n}=\left\{u: \exists t<\tau(2 n) \text { s.t. }\left\|u_{t}\right\|_{\infty}>n_{0}+n\right\},
$$

where $\tau$ is the stopping time from Eq.(4.4). By Tchebychev's inequality, by Proposition 4.4 and (2.6) we have

$$
\sum_{n=1}^{\infty} \mathbf{P}\left(\mathcal{E}_{n}\right) \leq \sum_{n=1}^{\infty} n^{-2} \sup _{t \leq \tau(2 n)} \mathbf{E}\left(\left\|u_{t}\right\|_{\infty}^{2}\right)<\infty .
$$

By the Borel-Cantelli Lemma, it means that almost surely only finitely many of the events $\mathcal{E}_{n}$ happen, and hence $\left\|u_{t}\right\|_{\infty}$ remains bounded as the cutoffs $R$ and $M$ in Eq.(4.5) are sent to infinity. Since $\tau(R)>0$ a.s. for all $R>1$ (by the a priori bound of Lemma 10.2) a uniform bound holds in a small interval of time and this can be iterated indefinitely. This implies uniform boundedness of $z_{t}$ and a similar argument holds for $\left\|z_{t}\right\|_{\infty}^{p}, p>1$. 
Proof of Theorem 4.1. By Proposition 4.4, it only remains to show that $\left\|\nabla^{m} u_{t}\right\|^{p}$ is bounded for $m>1$. Let $p=2$. We assume that it is true for $m-1$ and we consider

$$
\begin{aligned}
& \frac{1}{2} \mathrm{~d}\left\|\nabla^{m} u_{t}\right\|^{2} \\
& \leq-\frac{1}{2}\left\|\nabla^{m+1} u_{t}\right\|^{2} \mathrm{~d} t+\left(1+\frac{1}{2}\left(1+\alpha^{2}\right)\right)\left\|\nabla^{m} u_{t}\right\|^{2} \mathrm{~d} t \\
& \quad-\operatorname{Re}(1+\mathrm{i} \beta)\left(\nabla^{m} u_{t}, \nabla^{m}\left(\left|u_{t}\right|^{2 q} u_{t}\right)\right) \mathrm{d} t+\frac{1}{2}\left\|\nabla^{m} \xi_{t}\right\|^{2} \mathrm{~d} t+\operatorname{Re}\left(\nabla^{m} u_{t}, \nabla^{m} \xi_{t}\right) \mathrm{d} w_{t} \\
& \leq-\frac{1}{2}\left\|\nabla^{m+1} u_{t}\right\|^{2} \mathrm{~d} t+\left(C_{1}\left\|\nabla^{m} u_{t}\right\|^{2} \mathrm{~d} t+C_{2}\left\|u_{t}\right\|_{\mathrm{H}_{\delta, y}^{m-1}}^{2}+C_{3}\right) \mathrm{d} t \\
& \quad+(-1)^{m} \operatorname{Re}\left(u_{t}, \varphi^{-1} \nabla^{m}\left(\varphi \nabla^{m} \xi_{t}\right)\right) \mathrm{d} w_{t},
\end{aligned}
$$

Using (4.11) (with $u_{t}$ replaced by $\nabla^{m-1} u_{t}$ ), Proposition 4.5, and the recursion assumption, this can be bounded by:

$$
\frac{1}{2} \mathrm{~d}\left\|\nabla^{m} u_{t}\right\|^{2} \leq \frac{1}{2}\left(-\left\|\nabla^{m} u_{t}\right\|^{2}+C\right) \mathrm{d} t+(-1)^{m} \operatorname{Re}\left(u_{t}, \varphi^{-1} \nabla^{m}\left(\varphi \nabla^{m} \xi_{t}\right)\right) \mathrm{d} w_{t} .
$$

We then take expectations and integrate:

$$
\mathbf{E}\left\|\nabla^{m} u_{t}\right\|^{2} \leq \mathbf{E}\left\|\nabla^{m} u_{0}\right\|^{2}-\mathbf{E} \int_{0}^{t}\left(\left\|\nabla^{m} u_{s}\right\|^{2}-C\right) \mathrm{d} s .
$$

The case $p \neq 2$ is similar.

\section{Invariant Measures}

We now turn to the problem of the existence of an invariant measure for the process defined by Eq.(2.1). We construct here an explicit example of a smooth homogeneous random forcing which admits evident generalisations. Let $\xi(x)$ be a $\mathcal{C}^{\infty}$ almost periodic function on $\mathbf{R}^{d}$. Denoting $T_{y} \xi(x)=\xi(x+y)$, the set

$$
G=\overline{\left\{T_{y} \xi: y \in \mathbf{R}^{d}\right\}}{ }^{L^{\infty}}
$$

is a compact group which can be endowed with the normalised Haar measure $h$. We denote by $\left(G, \mathcal{F}_{1}, h\right)$ the corresponding probability space ( $\mathcal{F}_{1}$ is the sigma-algebra of Borel sets) and by $\xi_{y}$ the corresponding random variable. Let next $w_{\alpha}(t)$ be a standard Brownian motion (vanishing at 0$)$ on the probability space $\left(\mathcal{C}_{0}(\mathbf{R}, \mathbf{R}), \mathcal{F}_{2}, \mathbf{W}\right)\left(\mathcal{F}_{2}\right.$ is the sigma-algebra generated by the topology of uniform convergence on compact sets and $\mathbf{W}$ is the Wiener measure). Let $z_{\alpha}(t)=$ $y(t)-y(0)$ be a continuous process on $\left(G, \mathcal{F}_{1}, h\right)$ adapted to the filtration generated by the Brownian motion. We define the stochastic differential $\xi_{y(0)+z_{\alpha}(t)}(x) \mathrm{d} w_{\alpha}(t)$ on the probability space $(\Omega, \mathcal{F}, \mathbf{P})=\left(\mathcal{C}_{0}(\mathbf{R}, \mathbf{R}) \times G, \mathcal{F}_{2} \times \mathcal{F}_{1}, \mathbf{W} \times h\right)$. Let $\Xi_{\omega}(x, t)$ denote $\xi_{y(0)+z_{\alpha}(t)}(x) w_{\alpha}(t)$ where $\Omega \ni \omega=(\alpha, y(0)) \in \mathcal{C}_{0}(\mathbf{R}, \mathbf{R}) \times G$. By the nature of Haar measures, $\mathbf{P}$ is homogeneous in $x$, i.e. $T_{y}^{*} \mathbf{P}=\mathbf{P}$ for all $y \in \mathbf{R}^{d}$ (see Vishik and Fursikov [VF] for a discussion of homogeneous measures). 
Let $\Phi_{\omega}^{t}$ be the semi-flow generated by Eq.(2.1) with noise $\Xi_{\omega}(x, t)$. Using Lemma 10.3, we can define a Markov semi-group $\mathcal{P}_{t}$ acting on $\mathcal{C}_{\mathrm{b}}\left(\mathrm{L}_{\delta, 0}^{2}, \mathrm{C}\right)$ by

$$
\left(\mathcal{P}_{t} f\right)(u)=\int_{\mathrm{L}_{\delta, 0}^{2}} f(\eta) \mathbf{P}\left(\Phi_{\omega}^{t}(u) \in \mathrm{d} \eta\right) .
$$

$\mathcal{P}_{t}$ is a Markovian Feller semi-group (the Feller property follows from the continuity of $\Phi_{\omega}^{t}$ ). Its dual $\mathcal{P}_{t}^{*}$ acts on probability measures over $\mathrm{L}_{\delta, 0}^{2}$ by

$$
\left(\mathcal{P}_{t}^{*} \mu\right)(B)=\int_{\mathrm{L}_{\delta, 0}^{2}} \mathbf{P}\left(\Phi_{\omega}^{t}(u) \in B\right) \mu(\mathrm{d} u) .
$$

We call $\mu$ an invariant measure for Eq.(2.1) if $\mathcal{P}_{t}^{*} \mu=\mu$ for all $t>0$ (see Arnold [A]]).

In this section, we prove the following Theorem, which is actually a simple consequence of the bounds derived in Section 4 .

Theorem 5.1. There exists at least one invariant measure $\mu$ for Eq.(2.1). This measure is homogeneous in $x$ and its support is contained in $\bigcap_{m \geq 0} \mathrm{H}_{\mathrm{ul}}^{m}$.

Proof. We consider the family of measures $\left\{\bar{\mu}_{t}\right\}_{t>0}$, where

$$
\bar{\mu}_{t}=\frac{1}{t} \int_{0}^{t} \mathcal{P}_{s}^{*} \boldsymbol{\delta}_{0} \mathrm{~d} s
$$

$\boldsymbol{\delta}_{0}$ being the unit mass at $0 \in \mathrm{L}_{\delta, 0}^{2}$. By Theorem 4.1, this family is tight in $\mathrm{L}_{\delta, 0}^{2}$ for all $\delta>0$. Namely for any $\varepsilon>0$ there is a compact $K_{\varepsilon} \subset \subset \mathrm{L}_{\delta, 0}^{2}$ such that $\bar{\mu}_{t}\left(K_{\varepsilon}\right)>1-\varepsilon$. For the set $K_{\varepsilon}$ we choose the ball of radius $R(\varepsilon)$ in $\mathrm{H}_{\mathrm{ul}}^{m}(m>d / 2)$ for sufficiently large $R(\varepsilon)$ and the compactness follows from (2.5) and (2.7). By the Prokhorov Theorem, $\left\{\bar{\mu}_{t}\right\}_{t>0}$ is weakly precompact and thus there is at least one accumulation point $\mu$. By the standard Krylov-Bogolyubov argument $\mu$ is an invariant measure (see [ $\mathrm{Ar}, \overline{\mathrm{VF}}$, DZ2] for a detailed statement of these procedures).

Let $t_{n}, n=1,2, \ldots$ be a sequence such that $\mu=\mathrm{w}-\lim _{n \rightarrow \infty} \bar{\mu}_{t_{n}}$. Let $B_{R}$ be the ball of radius $R$ in $\mathrm{H}_{\delta, y}^{m}$ and let $f_{y, R}$ be any bounded continuous function on $\mathrm{L}_{\delta, y}^{2}$ vanishing on $B_{R}$ (which is a compact set). Since the topologies of $\mathrm{L}_{\delta, 0}^{2}$ and of $\mathrm{L}_{\delta, y}^{2}$ are equivalent, this function is continuous on $\mathrm{L}_{\delta, 0}^{2}$. Obviously $\left|\int f_{y, R}(\eta) \bar{\mu}_{t_{n}}(\mathrm{~d} \eta)\right|<\|f\|_{\infty} \varepsilon(R)$ for all $n$ and $y$, where $\varepsilon(R) \rightarrow 0$ as $R \rightarrow \infty$. By weak convergence of $\bar{\mu}_{t_{n}}$ to $\mu$ this also holds for $\mu$ and hence the support of $\mu$ must be contained in $\bigcap_{y \in \mathbf{R}} \mathrm{H}_{\delta, y}^{m}$.

We next prove the homogeneity of $\mu$. Let $f \in \mathcal{C}_{\mathrm{b}}\left(\mathrm{H}_{\mathrm{ul}}^{m}, \mathbf{C}\right)$ and define the translation operator $T_{y}$ by $T_{y} f(u)=f\left(T_{y} u\right)$. We have

$$
\begin{aligned}
\int T_{y} f(\eta) \mu(\mathrm{d} \eta) & =\lim _{n \rightarrow \infty} \frac{1}{t_{n}} \int_{0}^{t_{n}}\left(\int f\left(T_{y} \eta\right) \mathbf{P}\left(\Phi_{\omega}^{t}(0) \in \mathrm{d} \eta\right)\right) \mathrm{d} t \\
& =\lim _{n \rightarrow \infty} \frac{1}{t_{n}} \int_{0}^{t_{n}}\left(\int f(\eta) \mathbf{P}\left(T_{y}\left(\Phi_{\omega}^{t}(0)\right) \in \mathrm{d} \eta\right)\right) \mathrm{d} t \\
& =\lim _{n \rightarrow \infty} \frac{1}{t_{n}} \int_{0}^{t_{n}}\left(\int f(\eta) \mathbf{P}\left(\Phi_{T_{y} \omega}^{t}\left(T_{y}(0)\right) \in \mathrm{d} \eta\right)\right) \mathrm{d} t \\
& =\lim _{n \rightarrow \infty} \frac{1}{t_{n}} \int_{0}^{t_{n}}\left(\int f(\eta) \mathbf{P}\left(\Phi_{\omega}^{t}(0) \in \mathrm{d} \eta\right)\right) \mathrm{d} t=\int f(\eta) \mu(\mathrm{d} \eta)
\end{aligned}
$$


where we have used the homogeneity of $\mathbf{P}$. Since the above holds for all $f$, it proves that $\mu$ is homogeneous and the proof of Theorem 5.1 is finished.

Remark. In the above construction of a tight family of measures, we could have considered any homogeneous initial measure $\mu_{0}$ supported by $\cap_{m \geq 0} \mathrm{H}_{\mathrm{ul}}^{m}$ instead of $\boldsymbol{\delta}_{0}$.

\section{Entropy Estimates}

In this section, we define and estimate different notions of entropy for Eq.(2.1). We start with the topological entropy, then the measure-theoretic entropy and finally the $\varepsilon$-entropy. All these quantities are extensive, hence we actually define their spatial densities. We define the spatial density of upper box-counting dimension as well.

To do so we first introduce the basic dynamical setup: let $\Phi_{\omega}^{t}(t>0)$ be the solution semi-flow to Eq.(2.1) for given noise parameter $\omega$ and let $\theta^{t}$ be the shift semi-flow on $\Omega$ :

$$
\Xi_{\theta^{\tau} \omega}(x, t)=\Xi_{\omega}(x, t+\tau)-\Xi_{\omega}(x, t) .
$$

Let next $S^{t}$ be the semi-flow on $\mathrm{L}_{\delta, 0}^{2} \times \Omega$ defined by

$$
\begin{aligned}
S^{t}: \mathrm{L}_{\delta, 0}^{2} \times \Omega & \rightarrow \mathrm{L}_{\delta, 0}^{2} \times \Omega \\
(u, \omega) & \mapsto\left(\Phi_{\omega}^{t}(u), \theta^{t}(\omega)\right) .
\end{aligned}
$$

We consider the space $\mathrm{H}_{\mathrm{ul}}^{m}(m>d)$ endowed with the (weaker) topology of uniform convergence on the compact $Q \subset \subset \mathbf{R}^{d}$. By standard embeddings (see (2.6) and (2.7)) bounded sets of $\mathrm{H}_{\mathrm{ul}}^{m}$ are compact in $\mathrm{L}^{\infty}(Q)$. Following Crauel et al. [CDF], we define the random attractor $\mathcal{A}_{\omega}$ as follows: Let $B_{R}$ be the ball of radius $R$, centre 0 in $\mathrm{H}_{\mathrm{ul}}^{m}$ and let

$$
\begin{aligned}
& \mathcal{A}_{\omega}=\bar{\bigcup}_{R>0} \mathcal{A}(\omega, R)^{\mathrm{H}_{\mathrm{ul}}^{m}}, \\
& \mathcal{A}(\omega, R)=\bigcap_{T>0} \bigcup_{t>T} \Phi_{\theta^{-t} \omega}^{t}\left(B_{R}\right){ }^{\mathrm{H}_{\mathrm{ul}}} .
\end{aligned}
$$

By the estimates of Section $\sharp, \mathcal{A}_{\omega}$ is almost surely closed and bounded in $\mathrm{H}_{\mathrm{ul}}^{m}$ hence it is compact in $\mathrm{L}^{\infty}(Q)$ for any bounded $Q \subset \mathbf{R}^{d}$. Moreover the diameter of $\mathcal{A}_{\omega}$ in $\mathrm{H}_{\mathrm{ul}}^{m}$ is less than some $R_{\omega}$ with $\mathbf{P}\left(\omega: R_{\omega}<\infty\right)=1$ and $\mathbf{E}\left(\omega \mapsto R_{\omega}\right)<\infty$ (by Theorem 4.1). The following equivariance properties hold (we assume $\theta^{t} T_{x}=T_{x} \theta^{t}$ for all $(x, t) \in \mathbf{R}^{d} \times \mathbf{R}^{+}$):

$$
\begin{aligned}
\Phi_{\omega}^{t} \mathcal{A}_{\omega} & =\mathcal{A}_{\theta^{t} \omega}, \\
T_{x} \mathcal{A}_{\omega} & =\mathcal{A}_{T_{x} \omega},
\end{aligned}
$$

and it contains the support of any invariant measure for $S^{t}$. Let next $\mu$ be an invariant measure in the sense of Section 5, namely a stationary measure for the Markov semi-group (5.1). We also assume that $\mathbf{P}$ is an invariant measure for $\theta^{t}$. Then $\mu \times \mathbf{P}$ is an invariant measure for the 
dynamical system $\left(S^{t}, \mathcal{X}, \mathcal{B}\right)$ where $\mathcal{X}=\mathrm{L}_{\delta, 0}^{2} \times \Omega$ and $\mathcal{B}$ is the associated sigma-algebra. More precisely, one has

$$
\mathbf{E}\left(\omega \mapsto\left(\Phi_{\omega}^{t}\right)^{*} \mu\right)=\mu
$$

(which is only a rephrasing of $\mathcal{P}_{t}^{*} \mu=\mu$, see Eq.(5.2)). We introduce the following definitions:

Definition 6.1. Let $\tau>0, n \in \mathbf{N}$, and $Q \subset \subset \mathbf{R}$. We define a pseudo-metric $d_{\omega, n, \tau, Q}$ on $\mathrm{H}_{\mathrm{ul}}^{m}$ by

$$
d_{\omega, n, \tau, Q}(u, v)=\max _{k=0, \ldots, n-1}\left\|\Phi_{\omega}^{k \tau}(u)-\Phi_{\omega}^{k \tau}(v)\right\|_{L^{\infty}(Q)} .
$$

Let $N_{\omega, n, \tau, Q, \varepsilon}$ be the cardinality of a minimal $(n, \varepsilon)$-cover of $\left.\mathcal{A}_{\omega}\right|_{Q}$ (that is $N_{\omega, n, \tau, Q, \varepsilon}$ is the least number of open sets whose diameter in the metric $d_{\omega, n, \tau, Q}$ is at most $\varepsilon$ and whose union contains $\left.\mathcal{A}_{\omega}\right)$.

We define the cube $Q_{L}=\left[-\frac{1}{2} L, \frac{1}{2} L\right]^{d}$. We are now able to prove the existence of the spatial density of topological entropy $h_{\text {top }}$ for Eq.(2.1):

Proposition 6.2. For all $\tau>0$ the following limit exists:

$$
h_{\mathrm{top}}=\lim _{\varepsilon \rightarrow 0} \lim _{L \rightarrow \infty} \frac{1}{L^{d}} \lim _{n \rightarrow \infty} \frac{1}{n \tau} \int \log N_{\omega, n, \tau, Q_{L}, \varepsilon} \mathbf{P}(\mathrm{d} \omega) \text {, }
$$

This limit is independent of $\tau>0$.

Proof. The proof is similar to the deterministic case treated by Collet and Eckmann in [CE2] and is reproduced in Section 8 .

Let $\mathcal{U}=\left\{U_{1}, \ldots, U_{k}, \ldots\right\}$ be a countable (or finite) $\mu$-measurable partition of $\mathcal{A}_{\omega}$. For two partitions $\mathcal{U}$ and $\mathcal{V}$, we denote their refinement $\left\{U_{k} \cap V_{\ell}: U_{k} \in \mathcal{U}, V_{\ell} \in \mathcal{V}, \mu\left(U_{k} \cap V_{\ell}\right)>0\right\}$ by $\mathcal{U} \vee \mathcal{V}$. Moreover $\Phi_{\omega}^{-\tau}(\mathcal{U})=\left\{\Phi_{\omega}^{-\tau}\left(U_{k}\right): U_{k} \in \mathcal{U}\right\}$ is a measurable partition of $\mathcal{A}_{\theta^{-\tau} \omega}$ whenever $\mathcal{U}$ is a measurable partition of $\mathcal{A}_{\omega}$. (Here $\Phi_{\omega}^{-t}$ stands for the inverse of $\Phi_{\omega}^{t}$, namely $\Phi_{\omega}^{-t}(x)$ is the set of all pre-images of $x$.)

Definition 6.3. Let $H_{\mu}(\mathcal{U})$ and $H_{\mu}(\mathcal{U} \mid \mathcal{V})$ denote the entropy of a partition and the conditional entropy, both relative to a given measure $\mu$. They are defined as follows

$$
\begin{aligned}
H_{\mu}(\mathcal{U}) & =-\sum_{U \in \mathcal{U}} \mu(U) \log \mu(U) \\
H_{\mu}(\mathcal{U} \mid \mathcal{V}) & =-\sum_{U \in \mathcal{U}, V \in \mathcal{V}} \mu(U \cap V) \log \left(\frac{\mu(U \cap V)}{\mu(V)}\right) .
\end{aligned}
$$

We adopt here the convention $0 \log 0=0$ therefore $0<H_{\mu}(\mathcal{U}) \leq \log \operatorname{card}(\mathcal{U})$ (which is possibly infinite for countable $\mathcal{U}$ ). We also choose an arbitrary sequence $\Sigma_{\omega, \varepsilon}$ of partitions of $\mathcal{A}_{\omega}$ in sets of diameter at most $\varepsilon$ in the metric of $\mathrm{L}^{\infty}\left(Q_{1}\right)$. 
The second result in this section is the existence of the spatial density of measure-theoretic entropy $h_{\mu}$

Proposition 6.4. For all $\tau>0$ the following limit exists:

$$
h_{\mu}=\lim _{\varepsilon \rightarrow 0} \lim _{L \rightarrow \infty} \frac{1}{L^{d}} \lim _{n \rightarrow \infty} \frac{1}{n \tau} \int H_{\mu}\left(\bigvee_{x \in \mathbf{Z}^{d} \cap Q_{L}} \bigvee_{k=0}^{n-1} \Phi_{\omega}^{-k \tau} T_{-x}\left(\Sigma_{\theta^{k \tau} T_{x} \omega, \varepsilon}\right)\right) \mathbf{P}(\mathrm{d} \omega) .
$$

It is independent of $\tau>0$ and of the particular choice of the sequence of partitions $\Sigma_{\omega, \varepsilon}$.

Proof. Again, the proof is quite standard, see e.g. [KH, [LQ] or Section 9 .

We next introduce the notions of $\varepsilon$-entropy $\mathcal{H}_{\varepsilon}$ of Kolmogorov and Tikhomirov [ [KT] and of upper density of dimension $d_{\mathrm{up}}$.

Definition 6.5. Let $M_{\varepsilon, Q, \omega}$ be the least cardinality of an open cover of $\mathcal{A}_{\omega}$ by sets of diameter less than $\varepsilon$ in the metric of $\mathrm{L}^{\infty}(Q)$ where $Q$ is compact (we call this an $\varepsilon$-cover of $\left.\mathcal{A}_{\omega}\right|_{Q}$ ). Let $\mathcal{H}_{\varepsilon}$ be the Kolmogorov-Tikhomirov $\varepsilon-e n t r o p y$ defined by

$$
\mathcal{H}_{\varepsilon}=\lim _{L \rightarrow \infty} \int \frac{\log M_{\varepsilon, Q_{L}, \omega}}{L^{d}} \mathbf{P}(\mathrm{d} \omega)
$$

et let $d_{\mathrm{up}}(\omega)$ be the upper density of dimension of $\mathcal{A}_{\omega}$ :

$$
d_{\text {up }}=\limsup _{\varepsilon \rightarrow 0} \frac{\mathcal{H}_{\varepsilon}}{\log \varepsilon^{-1}}
$$

The main results of the section are the following inequalities involving the different entropies just defined. Corresponding inequalities in finite dimensional dynamical systems are well-known [KH].

Theorem 6.6. There is a $\gamma<\infty$ such that

$$
h_{\mu} \leq h_{\mathrm{top}} \leq \gamma d_{\mathrm{up}}<\infty \text {. }
$$

Before giving the proof of Theorem 6.6, we state a lemma which will prove useful later on.

Lemma 6.7. There are $C, \gamma$ such that for all (sufficiently large) $L$ and all (sufficiently small) $\varepsilon>0$, if $\|u-v\|_{L^{\infty}\left(Q_{L}\right)} \leq \varepsilon$ then for $t>0, L^{\prime}=L-C(1+t) \log 1 / \varepsilon$, one has

$$
\left\|\Phi_{\omega}^{t}(u)-\Phi_{\omega}^{t}(v)\right\|_{L^{\infty}\left(Q_{L^{\prime}}\right)} \leq C e^{\gamma t} \varepsilon
$$

almost surely. 
Proof. Let $u_{t}$ and $v_{t}$ be two solutions to Eq.(2.1). By Lemma 10.3,

$$
\left\|u_{t}-v_{t}\right\|_{\mathrm{L}_{\delta, 0}^{2}} \leq e^{\gamma t}\left\|u_{0}-v_{0}\right\|_{\mathrm{L}_{\delta, 0}^{2}}
$$

and moreover both $\left\|u_{t}\right\|_{\infty}$ and $\left\|v_{t}\right\|_{\infty}$ are bounded uniformly in time (see Proposition 4.5). Let $K_{t}(\cdot)$ be the convolution kernel associated with the semi-group $\exp (t \mathcal{L})\left(\operatorname{see}(4.1)\right.$ ) and let $r_{s}=$ $u_{s}-v_{s}$. By Duhamel's formula,

$$
\begin{aligned}
\left|r_{t}(x)\right| \leq & \left|K_{t} \star r_{0}(x)\right|+\left|\int_{0}^{t} K_{t-s} \star\left(\mathcal{G}_{1}\left(u_{s}, v_{s}\right) r_{s}+\mathcal{G}_{2}\left(u_{s}, v_{s}\right) \bar{r}_{s}\right)(x) \mathrm{d} s\right| \\
\leq & c_{1} e^{\gamma t}\left(\varepsilon+\sup _{|x-y|^{2} \leq C t \log 1 / \varepsilon}\left|r_{0}(y)\right|\right) \\
& \quad+\sup _{0 \leq s \leq t}\left(\left\|\mathcal{G}_{1}\left(u_{s}, v_{s}\right)\right\|_{\infty}+\left\|\mathcal{G}_{2}\left(u_{s}, v_{s}\right)\right\|_{\infty}\right) \int_{0}^{t} \frac{\left|K_{t-s}\right|}{\sqrt{\varphi_{\delta, 0}}} \star\left(\sqrt{\varphi_{\delta, x}}\left|r_{s}\right|\right)(x) \mathrm{d} s \\
\leq & c_{1} e^{\gamma t}\left(\varepsilon+\sup _{|x-y|^{2} \leq C t \log 1 / \varepsilon}\left|r_{0}(y)\right|\right)+c_{2}\left\|\sqrt{\varphi_{\delta, x}}\left|r_{0}\right|\right\|_{2} \int_{0}^{t} e^{\gamma s} \| \frac{\left|K_{t-s}\right|}{\sqrt{\varphi \delta, 0}}||_{2} \mathrm{~d} s \\
\leq & c_{3}(1+t) e^{(1+\gamma) t}\left(2 \varepsilon+\sup _{|x-y|^{2} \leq C t \log 1 / \varepsilon}\left|r_{0}(y)\right|+\sup _{|x-y| \leq C \log 1 / \varepsilon}\left|r_{0}(y)\right|\right) \\
\leq & 4 c_{3} e^{(2+\gamma) t} \varepsilon, \quad
\end{aligned}
$$

where in the last line we have assumed $|x| \leq \frac{1}{2} L-C(1+t) \log 1 / \varepsilon$ (hence $|y| \leq \frac{1}{2} L$ ) and used the assumption $\sup _{|y| \leq L / 2}\left|r_{0}(y)\right| \leq \varepsilon$.

Proof of Theorem 6.6. We split Theorem 6.6 into three independent statements, namely each one of the three inequalities in (6.5).

Proof of $h_{\mu} \leq h_{\mathrm{top}}$. We follow the most standard proof (originally by Misiurewicz, quoted in [KH]). We modify the partition $\Sigma_{\omega, \varepsilon}=\left\{\sigma_{1}, \ldots, \sigma_{N}\right\}$ by "shrinking" each element, namely by replacing each $\sigma_{k}$ by a closed set $U_{k}$ with $U_{k} \subset \sigma_{k}$ and we define $U_{0}=\mathcal{A}_{\omega} \backslash \cup_{k=1}^{N} U_{k}$. We thus obtain a new partition $\mathcal{U}_{\omega, \varepsilon}=\left\{U_{0}, \ldots, U_{N}\right\}$ and an open cover $\mathcal{V}_{\omega, \varepsilon}=\left\{U_{1} \cup U_{0}, \ldots, U_{N} \cup U_{0}\right\}$. We assume that the $U_{k}$ have been chosen such that $\int H_{\mu}\left(\Sigma_{\omega, \varepsilon} \mid \mathcal{U}_{\omega, \varepsilon}\right) \mathbf{P}(\mathrm{d} \omega)<1$. Remark that

$$
\operatorname{card}\left(\bigvee_{x \in \mathbf{Z}^{d} \cap Q_{L}} \bigvee_{j=0}^{n-1} \Phi_{\omega}^{-j \tau} T_{-x}\left(\mathcal{U}_{\theta^{-j \tau}} T_{x} \omega, \varepsilon\right)\right) \leq 2^{n L^{d}} \operatorname{card}\left(\bigvee_{x \in \mathbf{Z}^{d} \cap Q_{L}} \bigvee_{j=0}^{n-1} \Phi_{\omega}^{-j \tau} T_{-x}\left(\mathcal{V}_{\theta^{-j \tau} T_{x} \omega, \varepsilon}\right)\right)
$$

and by Definition 6.3

$$
\begin{aligned}
& H_{\mu}\left(\bigvee_{x \in \mathbf{Z}^{d} \cap Q_{L}} \bigvee_{j=0}^{n-1} \Phi_{\omega}^{-j \tau} T_{-x}\left(\mathcal{U}_{\theta^{-j \tau} T_{x} \omega, \varepsilon}\right)\right) \\
& \leq \log \operatorname{card}\left(\bigvee_{x \in \mathbf{Z}^{d} \cap Q_{L}} \bigvee_{j=0}^{n-1} \Phi_{\omega}^{-j \tau} T_{-x}\left(\mathcal{U}_{\theta^{-j \tau} T_{x} \omega, \varepsilon}\right)\right) \\
& \leq \log \operatorname{card}\left(\bigvee_{x \in \mathbf{Z}^{d} \cap Q_{L}} \bigvee_{j=0}^{n-1} \Phi_{\omega}^{-j \tau} T_{-x}\left(\mathcal{V}_{\theta^{-j \tau} T_{x} \omega, \varepsilon}\right)\right)+n L^{d} \log 2 .
\end{aligned}
$$


Consequently

$$
\begin{aligned}
& \lim _{L \rightarrow \infty} \frac{1}{L^{d}} \lim _{n \rightarrow \infty} \frac{1}{n \tau} \int H_{\mu}\left(\bigvee_{x \in \mathbf{Z}^{d} \cap Q_{L}} \bigvee_{j=0}^{n-1} \Phi_{\omega}^{-j \tau} T_{-x}\left(\mathcal{U}_{\theta^{-j \tau} T_{x} \omega, \varepsilon}\right)\right) \mathbf{P}(\mathrm{d} \omega) \\
& \leq \lim _{L \rightarrow \infty} \frac{1}{L^{d}} \lim _{n \rightarrow \infty} \frac{1}{n \tau} \int \log \operatorname{card}\left(\bigvee_{x \in \mathbf{Z}^{d} \cap Q_{L}} \bigvee_{j=0}^{n-1} \Phi_{\omega}^{-j \tau} T_{-x}\left(\mathcal{V}_{\theta^{-j \tau} T_{x} \omega, \varepsilon}\right)\right) \mathbf{P}(\mathrm{d} \omega)+\frac{\log C}{\tau} .
\end{aligned}
$$

Moreover, the difference between the original partition and the new one is small, namely:

$$
\begin{aligned}
& \lim _{n \rightarrow \infty} \frac{1}{n \tau} \int H_{\mu}\left(\bigvee_{j=0}^{n-1} \Phi_{\omega}^{j \tau}\left(\Sigma_{\theta^{-j \tau} \omega, \varepsilon}\right)\right) \mathbf{P}(\mathrm{d} \omega) \\
& \leq \lim _{n \rightarrow \infty} \frac{1}{n \tau} \int H_{\mu}\left(\bigvee_{j=0}^{n-1} \Phi_{\omega}^{j \tau}\left(\mathcal{U}_{\theta^{-j \tau} \omega, \varepsilon}\right)\right) \mathbf{P}(\mathrm{d} \omega)+\frac{1}{\tau} \int H_{\mu}\left(\Sigma_{\omega, \varepsilon} \mid \mathcal{U}_{\omega, \varepsilon}\right) \mathbf{P}(\mathrm{d} \omega)
\end{aligned}
$$

Since all the above holds for arbitrarily large $\tau>0$ we get

$$
h_{\mu} \leq \lim _{\varepsilon \rightarrow 0} \lim _{L \rightarrow \infty} \frac{1}{L^{d}} \lim _{n \rightarrow \infty} \frac{1}{n \tau} \int \log \operatorname{card}\left(\bigvee_{x \in \mathbf{Z}^{d} \cap Q_{L}} \bigvee_{j=0}^{n-1} \Phi_{\omega}^{-j \tau} T_{-x}\left(\mathcal{V}_{\theta^{-j \tau} T_{x} \omega, \varepsilon}\right)\right) \mathbf{P}(\mathrm{d} \omega)
$$

Let next $\delta_{\omega, \varepsilon}$ be the Lebesgue number of the cover $\mathcal{V}_{\omega, \varepsilon}$ (namely the largest $\delta_{\omega, \varepsilon}>0$ such that every ball of diameter $\delta_{\omega, \varepsilon}$ is contained in an element of $\mathcal{V}_{\omega, \varepsilon}$ ). Indeed $\delta_{\omega, \varepsilon}$ is also the Lebesgue number of $\bigvee_{j=0}^{n-1} \Phi_{\omega}^{j \tau}\left(\mathcal{V}_{\theta^{-j \tau} \omega, \varepsilon}\right)$ with respect to the metric $d_{\omega, n, \tau, Q_{L}}$. Hence

$$
\operatorname{card}\left(\bigvee_{x \in \mathbf{Z}^{d} \cap Q_{L}} \bigvee_{j=0}^{n-1} \Phi_{\omega}^{-j \tau} T_{-x}\left(\mathcal{V}_{\theta^{-j \tau} T_{x} \omega, \varepsilon}\right)\right) \leq M_{\delta_{\omega, \varepsilon}, Q_{L}, \omega}
$$

and this proves that the r.h.s. of (6.6) is less than $h_{\mathrm{top}}$.

Proof of $h_{\text {top }} \leq \gamma d_{\text {up }}$. The proof follows [CE2]. Let $\rho>0$ be such that $\mathcal{H}_{\varepsilon} \leq\left(d_{\text {up }}+\rho\right) \log 1 / \varepsilon$ for all $\varepsilon<\varepsilon_{0}$ and then let $L_{0}=L_{0}(\varepsilon, \rho)$ be such that all $L>L_{0}$ yield

$$
\left|\int \frac{\log M_{\varepsilon, Q_{L}, \omega}}{L^{d}} \mathbf{P}(\mathrm{d} \omega)-\mathcal{H}_{\varepsilon}\right| \leq \rho .
$$

Let $L^{\prime}=L+C(T+1) \log (1 / \varepsilon)$ and $\varepsilon^{\prime}=C^{-1} \exp (-\gamma T) \varepsilon$ (see Lemma 6.7). Let an $\varepsilon^{\prime}$-cover of $\left.\mathcal{A}_{\omega}\right|_{Q_{L^{\prime}}}$ (in the sense of Definition 6.5) be given. Then it is also a $(T / \tau, \varepsilon)$-cover (in the sense of Definition 6.1), hence

$$
N_{\omega, T / \tau, \tau, Q_{L}, \varepsilon} \leq M_{\varepsilon^{\prime}, Q_{L^{\prime}}, \omega}
$$


from which follows

$$
\begin{aligned}
h_{\mathrm{top}} & =\lim _{\varepsilon \rightarrow 0} \lim _{L \rightarrow \infty} \frac{1}{L^{d}} \lim _{T \rightarrow \infty} \frac{1}{T} \int \log N_{\omega, T / \tau, \tau, Q_{L}, \varepsilon} \mathbf{P}(\mathrm{d} \omega) \\
& =\lim _{\varepsilon \rightarrow 0} \lim _{L \rightarrow \infty} \frac{1}{L^{d}} \inf _{T} \frac{1}{T} \int \log N_{\omega, T / \tau, \tau, Q_{L}, \varepsilon} \mathbf{P}(\mathrm{d} \omega) \\
& \leq \lim _{\varepsilon \rightarrow 0} \lim _{L \rightarrow \infty} \frac{1}{T} \int \frac{\log M_{\varepsilon^{\prime}, Q_{L^{\prime}, \omega}}}{L^{d}} \mathbf{P}(\mathrm{d} \omega) \\
& \leq \lim _{\varepsilon \rightarrow 0} \lim _{L \rightarrow \infty} \frac{1}{T}\left(\left(d_{\mathrm{up}}+\rho\right) \log 1 / \varepsilon^{\prime}+\rho\right) .
\end{aligned}
$$

Since $\log 1 / \varepsilon^{\prime}=\gamma T+\log (C / \varepsilon)$, the limit $T \rightarrow \infty$ and $\rho \rightarrow 0$ leaves only $\gamma d_{\text {up }}$ on the r.h.s. above.

Proof of $d_{\text {up }}<\infty$. We want to prove a bound on $\mathcal{H}_{\varepsilon}$ of the form $\mathcal{H}_{\varepsilon} \leq C \log 1 / \varepsilon$ for small $\varepsilon>0$. To do so we use iteratively the following bound:

Lemma 6.8. There are $A, B_{\omega}, C>0$ such that for all $L>0$ and sufficiently small $\varepsilon>0$, one has almost surely

$$
M_{\varepsilon, Q_{L}, \omega} \leq M_{2 \varepsilon, Q_{L+C}, \theta^{-1} \omega} A^{L^{d}} B_{\theta^{-1} \omega}^{1 / \varepsilon^{2}} .
$$

The proof of Lemma 6.8 is postponed to Section 7 . Let $\varepsilon>0, L>0$. Remember that there is an $R_{\omega}$ such that $M_{R_{\omega}, Q_{L}, \omega}=1$. Let $T_{\omega}$ be the smallest integer larger than $(\log 2)^{-1} \log \left(R_{\omega} / \varepsilon\right)$. By iterating $T_{\omega}$ times the bound (6.7), we obtain

$$
M_{\varepsilon, Q_{L}, \omega} \leq \prod_{n=1}^{T_{\omega}} A^{(L+(n-1) C)^{d}} B_{\theta^{-n} \omega}^{1 / \varepsilon^{2}}
$$

hence

$$
\mathcal{H}_{\varepsilon}=\lim _{L \rightarrow \infty} \int \frac{\log M_{\varepsilon, Q_{L}, \omega}}{L^{d}} \mathbf{P}(\mathrm{d} \omega) \leq \mathbf{E}\left(\omega \mapsto T_{\omega}\right) \log A \leq C\left(\log 1 / \varepsilon+\log \mathbf{E}\left(\omega \mapsto R_{\omega}\right)\right),
$$

(by Jensen's inequality) or, by Definition 6.5,

$$
d_{\mathrm{up}}=\limsup _{\varepsilon \rightarrow 0} \frac{\mathcal{H}_{\varepsilon}}{\log 1 / \varepsilon} \leq C,
$$

which is the bound we wanted to prove. With this inequality, the proof of Theorem 6.6 is finished. 


\section{Proof of Lemma 6.8}

We give the proof for the notationally convenient case $d=1$. Let $u$ and $v$ be two orbits of Eq.(2.1) with initial conditions $u_{0}$ and $v_{0}$ such that $u_{0}$ and $v_{0}$ belong to $\mathcal{A}_{\omega}$. The difference $r=u-v$ satisfies almost surely the equation

$$
\partial_{t} r=\left(1+(1+\mathrm{i} \alpha) \partial_{x}^{2}\right) r+\mathcal{G}_{1}(u, v) r+\mathcal{G}_{2}(u, v) \bar{r},
$$

where we have used the notation of Eq.(10.1).

Let $\chi(x)$ be a smooth and monotone function satisfying $\chi(x)=1$ if $x \leq 1$ and $\chi(x)=0$ if $x \geq 2$. We decompose the kernel of $\exp (t \mathcal{L})$ into a low frequency part and a high frequency part:

$$
\begin{aligned}
& K_{t}^{(-)}(x)=\frac{1}{2 \pi} \int_{-\infty}^{\infty} e^{\mathrm{i} p x+t\left(1-(1+\mathrm{i} \alpha) p^{2}\right)} \chi\left(\left|p / p^{*}\right|\right) \mathrm{d} p \\
& K_{t}^{(+)}(x)=\frac{1}{2 \pi} \int_{-\infty}^{\infty} e^{\mathrm{i} p x+t\left(1-(1+\mathrm{i} \alpha) p^{2}\right)}\left(1-\chi\left(\left|p / p^{*}\right|\right)\right) \mathrm{d} p
\end{aligned}
$$

where $p^{*}>4$ is a sufficiently large real number. We decompose the solutions $r_{t}(x)$ to Eq.(7.1) accordingly:

$$
\begin{aligned}
r_{t}(x) & =r_{t}^{(-)}(x)+r_{t}^{(+)}(x) \\
r_{t}^{(-)}(x) & =\left(K_{t}^{(-)} \star r_{0}\right)(x)+\int_{0}^{t}\left(K_{t-s}^{(-)} \star\left(\mathcal{G}_{1}\left(u_{s}, v_{s}\right) r_{s}+\mathcal{G}_{2}\left(u_{s}, v_{s}\right) \bar{r}_{s}\right)\right)(x) \mathrm{d} s, \\
r_{t}^{(+)}(x) & =\left(K_{t}^{(+)} \star r_{0}\right)(x)+\int_{0}^{t}\left(K_{t-s}^{(+)} \star\left(\mathcal{G}_{1}\left(u_{s}, v_{s}\right) r_{s}+\mathcal{G}_{2}\left(u_{s}, v_{s}\right) \bar{r}_{s}\right)\right)(x) \mathrm{d} s
\end{aligned}
$$

The kernels $K_{t}^{(-)}$and $K_{t}^{(+)}$have some regularity and decay properties that we next describe: let the Bernstein class $B_{R, k}$ be the following set of functions:

$$
B_{R, k} \equiv\left\{f \in \mathrm{L}^{\infty}: f \text { extends to an entire function, }|f(z)| \leq R e^{k|\operatorname{Im} z|}\right\} .
$$

We have

Lemma 7.1. For all $p^{*}>4, t>\frac{1}{2}, f \in \mathrm{L}^{\infty}, K_{t}^{(-)} \star f$ is in $B_{R, 2 p^{*}}$ with $R \leq 2 C_{0}\|f\|_{\infty}$. Moreover, for all $n \in \mathrm{N}$, there is a $C_{n}>0$ such that

$$
\begin{aligned}
& \left|K_{t}^{(-)}(x)\right| \leq \frac{C_{n}}{\sqrt{t}}\left(1+x^{2} / t\right)^{-n} \\
& \left|K_{t}^{(+)}(x)\right| \leq \frac{C_{n}}{\sqrt{t}} e^{-\left(p^{*}\right)^{2} t / 2}\left(1+x^{2} / t\right)^{-n} .
\end{aligned}
$$

The proof of Lemma 7.1 is omitted, see [CE2, Ro]. 
Pick a $2 \varepsilon$-cover of $\left.\mathcal{A}_{\omega}\right|_{Q_{L+C(\varepsilon)}}$ (which exists a.s. by compactness, see Definition 6.5) and let $u$ and $v$ belong to one of its elements. Then $r_{0}=u-v$ satisfies $\left|r_{0}(x)\right| \leq 2 \varepsilon$ for $|x| \leq \frac{1}{2}(L+C(\varepsilon))$. Define

$$
\xi_{y}^{(n)}(x)=\frac{1}{\left(1+(x-y)^{2}\right)^{n / 2}} .
$$

Remark that Lemma 10.3 also holds with $\varphi_{y}$ replaced by $\xi_{y}^{(n)}(n \geq 2)$. Moreover by reproducing the proof of Lemma 6.7 using the bounds from Lemma 7.1 we obtain (for $|x| \leq L / 2$ ):

$$
\begin{aligned}
\left|r_{1}^{(-)}(x)\right| & \leq\left|K_{1}^{(-)} \star r_{0}(x)\right|+C \int_{0}^{1}\left\|K_{1-s}^{(-)} / \sqrt{\xi_{0}^{(n)}}\right\|_{2}\left\|\sqrt{\xi_{y}^{(n)}} r_{s}\right\|_{2} \\
& \leq C \varepsilon+2 C \varepsilon \int_{0}^{1} \frac{C_{n}}{\sqrt{1-s}} e^{\gamma s} \mathrm{~d} s \\
& \leq A \varepsilon
\end{aligned}
$$

where $A$ depends on $n$ but not on $p^{*}$ and

$$
\begin{aligned}
\left|r_{1}^{(+)}(x)\right| & \leq\left|K_{1}^{(+)} \star r_{0}(x)\right|+C \int_{0}^{1}\left\|K_{1-s}^{(+)} / \sqrt{\xi_{0}^{(n)}}\right\|_{2}\left\|\sqrt{\xi_{y}^{(n)}} r_{s}\right\|_{2} \\
& \leq e^{-\left(p^{*}\right)^{2} / 2} \varepsilon+2 C \varepsilon \int_{0}^{1} \frac{C_{n} e^{-\left(p^{*}\right)^{2}(1-s) / 2}}{\sqrt{1-s}} e^{\gamma s} \mathrm{~d} s \\
& \leq B\left(p^{*}\right) \varepsilon
\end{aligned}
$$

where $B\left(p^{*}\right) \rightarrow 0$ as $p^{*} \rightarrow \infty$. We choose $p^{*}$ so large that $B\left(p^{*}\right)<\frac{1}{2}$.

We next use a result of Cartwright (see [KT], Eq.(191)): for all $f$ in the Bernstein class $B_{R, 2 p^{*}}$ (see (7.2)), the following identity holds:

$$
f(x)=\frac{\sin \left(8 p^{*} x\right)}{32\left(p^{*}\right)^{2}} \sum_{n=-\infty}^{\infty}(-1)^{n} f\left(x_{n}\right) \frac{\sin \left(4 p^{*}\left(x-x_{n}\right)\right)}{\left(x-x_{n}\right)^{2}}
$$

where $x_{n}=\frac{n \pi}{8 p^{*}}$. Let $f, g$ be in $B_{R, 2 p^{*}}$. A simple application of Eq.(7.5) shows that

$$
\|f-g\|_{L^{\infty}\left(Q_{L}\right)} \leq C \sup _{|n| \leq\left[4 p^{*} L / \pi\right]+4 C p^{*} /(\varepsilon \pi)}\left|f\left(x_{n}\right)-g\left(x_{n}\right)\right|+\frac{1}{4} \varepsilon .
$$

Hence, among all the functions in $B_{R_{\omega}, 2 p^{*}}$ that are bounded by $A \varepsilon$ in $\left[-\frac{1}{2} L, \frac{1}{2} L\right]$ (by $(7.3), r_{1}^{(-)}$ is such a function), at most $(4 A)^{C p^{*} L}\left(4 R_{\omega} / \varepsilon\right)^{C p^{*} / \varepsilon}$ of them are $\varepsilon / 2-$ separated on $Q_{L}$. By taking a ball of diameter $\varepsilon$ around each of them, and repeating the operation for each element of the original $2 \varepsilon$-cover, we get an $\varepsilon$-cover of $\left.\Phi_{\omega}^{1}\left(\mathcal{A}_{\omega}\right)\right|_{Q_{L}}=\left.\mathcal{A}_{\theta^{1} \omega}\right|_{Q_{L}}$. The number of elements in this cover is at most

$$
(4 A)^{C p^{*} L}\left(4 R_{\omega} / \varepsilon\right)^{C p^{*} / \varepsilon} M_{2 \varepsilon, Q_{L+C}, \omega} .
$$

The proof of Lemma 6.8 is complete. 


\section{Proof of Proposition 6.2}

We follow Collet and Eckmann's proof [CE2], which is itself an adaptation of standard proofs of existence of the topological entropy, see e.g. [KH] and references therein. The proof of Proposition 6.2 is based on the following inequalities:

Lemma 8.1. For all compacts $Q, Q^{\prime}$, all $m, n \in \mathbf{N}$ and $\varepsilon>\varepsilon^{\prime}>0$ one has

$$
\begin{aligned}
N_{\omega, n, \tau, Q, \varepsilon} & \leq N_{\omega, n, \tau, Q, \varepsilon^{\prime}}, \\
N_{\omega, n, \tau, Q \cup Q^{\prime}, \varepsilon} & \leq N_{\omega, n, \tau, Q, \varepsilon} N_{\omega, n, \tau, Q^{\prime}, \varepsilon}, \\
N_{\omega, n+m, \tau, Q, \varepsilon} & \leq N_{\omega, n, \tau, Q, \varepsilon} N_{\theta^{n \tau} \omega, m, \tau, Q, \varepsilon},
\end{aligned}
$$

Furthermore for any $\tau^{\prime}<\tau$ the following inequalities hold:

$$
N_{\omega, n, \tau^{\prime}, Q_{L}, \varepsilon} \leq N_{\omega, n, \tau, Q_{f(L)}, g(\varepsilon)} \leq N_{\omega, n, \tau^{\prime}, Q_{f(f(L))}, g(g(\varepsilon))}
$$

where $f(L)=L+C(\tau+1) \log \varepsilon^{-1}$ and $g(\varepsilon)=c \exp (-\gamma \tau) \varepsilon$ with $C, c, \gamma$ some constants.

Lemma 8.1 implies immediately that the limit in Eq.(6.3) exists: by subadditivity (8.3) and by invariance of $\mathbf{P}$ under $\theta^{t}$, we get that

$$
\Lambda_{1}=\lim _{n \rightarrow \infty} \frac{1}{n \tau} \int \log N_{\omega, n, \tau, Q_{L}, \varepsilon} \mathbf{P}(\mathrm{d} \omega)
$$

exists, it is non-increasing in $\varepsilon$ and by further subadditivity (8.2)

$$
\Lambda_{2}=\lim _{L \rightarrow \infty} \frac{1}{L^{d}} \Lambda_{1}
$$

also exists and is non-increasing in $\varepsilon$ (by (8.1)). Hence the limit in Eq.(6.3) exists. By (8.4), it is independent of $\tau$.

Proof of Lemma 8.1. The inequality (8.1) is obvious from the definitions. We prove (8.2) by making the observation that if $\left\{A_{1}, \ldots, A_{N}\right\}$ is an $(n, \varepsilon)$-cover of $\left.\mathcal{A}_{\omega}\right|_{Q}$ and $\left\{B_{1}, \ldots, B_{M}\right\}$ an $(n, \varepsilon)$-cover of $\left.\mathcal{A}_{\omega}\right|_{Q^{\prime}}$, then $\left\{A_{j} \cap B_{k}: j=1, \ldots, N, k=1, \ldots, M\right\}$ is an $(n, \varepsilon)$-cover of $\left.\mathcal{A}_{\omega}\right|_{Q \cup Q^{\prime}}$.

Similarly if $\left\{A_{1}, \ldots, A_{N}\right\}$ is an $(n, \varepsilon)$-cover of $\left.\mathcal{A}_{\omega}\right|_{Q}$ and $\left\{B_{1}, \ldots, B_{M}\right\}$ an $(m, \varepsilon)$-cover of $\left.\mathcal{A}_{\theta^{n \tau} \omega}\right|_{Q}$, then $\left\{A_{j} \cap \Phi_{\omega}^{-n \tau} B_{k}: j=1, \ldots, N, k=1, \ldots, M\right\}$ is an $(m+n, \varepsilon)$-cover of $\left.\mathcal{A}_{\omega}\right|_{Q}$ which proves (8.3).

The inequality (8.4) follows immediately from Lemma 6.7, since if $D$ is a set of diameter $g(\varepsilon)$ in the metric $d_{\omega, n, \tau, Q_{f(L)}}$ then $D$ is a set of diameter at most $\varepsilon$ in the metric $d_{\omega, n, \tau^{\prime}, Q_{L}}$.

Remark. The topology of $\mathrm{L}^{\infty}(Q)$ is a simplifying choice (as far as Eq.(8.2) is concerned), but [CE3] have demonstrated that other topologies can be used as well. 


\section{Proof of Proposition 6.4}

This proof is, like the proof of Proposition 6.2, based on subadditive bounds. We use wellknown properties of the function $H_{\mu}(\cdot)$, see $[\overline{\mathrm{KH}}]$, Chapter 4.3 (in particular Proposition 4.3.3). We recall that $x \mapsto-x \log x$ is concave, hence for any partition $\mathcal{U}$ and any $t>0$, the following holds:

$$
\int H_{\mu}\left(\Phi_{\omega}^{-t}(\mathcal{U})\right) \mathbf{P}(\mathrm{d} \omega) \leq H_{\mu}\left(\int \Phi_{\omega}^{-t}(\mathcal{U}) \mathbf{P}(\mathrm{d} \omega)\right)=H_{\mu}(\mathcal{U})
$$

where we have used Eq.(6.2). We thus have

$$
\begin{aligned}
& \int H_{\mu}\left(\bigvee_{k=0}^{n+m-1} \Phi_{\omega}^{-k \tau}\left(\Sigma_{\theta^{k \tau} \omega, \varepsilon}\right)\right) \mathbf{P}(\mathrm{d} \omega) \\
= & \int H_{\mu}\left(\bigvee_{k=0}^{n-1} \Phi_{\omega}^{-k \tau}\left(\Sigma_{\theta^{k \tau} \omega, \varepsilon}\right)\right) \mathbf{P}(\mathrm{d} \omega) \\
& +\int H_{\mu}\left(\bigvee_{k=n}^{n+m-1} \Phi_{\omega}^{-k \tau}\left(\Sigma_{\theta^{k \tau} \omega, \varepsilon}\right) \mid \bigvee_{k=0}^{n-1} \Phi_{\omega}^{-k \tau}\left(\Sigma_{\theta^{k \tau} \omega, \varepsilon}\right)\right) \mathbf{P}(\mathrm{d} \omega) \\
\leq & \int H_{\mu}\left(\bigvee_{k=0}^{n-1} \Phi_{\omega}^{-k \tau}\left(\Sigma_{\theta^{k \tau} \omega, \varepsilon}\right)\right) \mathbf{P}(\mathrm{d} \omega)+\int H_{\mu}\left(\Phi_{\omega}^{-n \tau} \bigvee_{k=0}^{m-1} \Phi_{\theta^{n \tau} \omega}^{-k \tau}\left(\Sigma_{\theta^{(k+n) \tau} \omega, \varepsilon}\right)\right) \mathbf{P}(\mathrm{d} \omega) \\
\leq & \int H_{\mu}\left(\bigvee_{k=0}^{n-1} \Phi_{\omega}^{-k \tau}\left(\Sigma_{\theta^{k \tau} \omega, \varepsilon}\right)\right) \mathbf{P}(\mathrm{d} \omega)+\iint H_{\mu}\left(\Phi_{\omega^{\prime}}^{-n \tau} \bigvee_{k=0}^{m-1} \Phi_{\omega}^{-k \tau}\left(\Sigma_{\theta^{k \tau} \omega, \varepsilon}\right)\right) \mathbf{P}\left(\mathrm{d} \omega^{\prime}\right) \mathbf{P}(\mathrm{d} \omega) \\
\leq & \int H_{\mu}\left(\bigvee_{k=0}^{n-1} \Phi_{\omega}^{-k \tau}\left(\Sigma_{\theta^{k \tau} \omega, \varepsilon}\right)\right) \mathbf{P}(\mathrm{d} \omega)+\int H_{\mu}\left(\bigvee_{k=0}^{m-1} \Phi_{\omega}^{-k \tau}\left(\Sigma_{\theta^{k \tau} \omega, \varepsilon}\right)\right) \mathbf{P}(\mathrm{d} \omega),
\end{aligned}
$$

namely subadditivity in the time variable. We can prove subadditivity in the space variable in a similar way. Thus the first two limits in Eq.(6.4) exist. These limits are monotonically increasing as $\varepsilon \rightarrow 0$, hence the third limit is well-defined.

We next prove that the limit is independent of the choice of $\Sigma_{\omega, \varepsilon}$ : let $\Sigma_{\omega, \varepsilon}$ and $\widetilde{\Sigma}_{\omega, \varepsilon}$ be two different sequences, we get (by the Rokhlin inequality)

$$
\begin{aligned}
& \lim _{L \rightarrow \infty} \frac{1}{L^{d}} \lim _{n \rightarrow \infty} \frac{1}{n \tau} H_{\mu}\left(\bigvee_{\substack{x \in \in \\
\mathbf{z}^{d} \cap Q_{L}}} \bigvee_{k=0}^{n-1} \Phi_{\omega}^{-k \tau} T_{-x}\left(\Sigma_{\theta^{k \tau} T_{x} \omega, \varepsilon}\right)\right) \\
& -\lim _{L \rightarrow \infty} \frac{1}{L^{d}} \lim _{n \rightarrow \infty} \frac{1}{n \tau} H_{\mu}\left(\bigvee_{\substack{x \in \\
\mathbf{z}^{d} \cap Q_{L}}} \bigvee_{k=0}^{n-1} \Phi_{\omega}^{-k \tau} T_{-x}\left(\widetilde{\Sigma}_{\theta^{k \tau}} T_{x} \omega, \varepsilon\right)\right) \\
& \leq H_{\mu}\left(\Sigma_{\omega, \varepsilon} \mid \widetilde{\Sigma}_{\omega, \varepsilon}\right)+H_{\mu}\left(\widetilde{\Sigma}_{\omega, \varepsilon} \mid \Sigma_{\omega, \varepsilon}\right)
\end{aligned}
$$

and the r.h.s. above vanishes as $\varepsilon \rightarrow 0$ since these sequences generate the whole sigma-algebra of $\mathcal{A}_{\omega}$ in this limit. 
We prove that Eq.(6.4) is independent of $\tau$ by using Lemma 6.7 and an argument similar to the one used in Section 8 .

\section{Uniqueness of Solutions}

In this section, we use the Contraction Mapping Principle to prove uniqueness of solutions to Eq.(4.5) as well as estimates on the stopping times Eq.(4.4) (along the lines of Da Prato and Zabczyk, [DZ1] Chapter 7). We define a Banach space $\mathcal{B}_{p, K, T}$ of complex-valued predictable processes $u$ on the time interval $[0, T]$ with norm defined by

$$
\|u\|_{p}=\left(\sup _{0 \leq t \leq T} \mathbf{E}\left(e^{-K t}\left\|u_{t}\right\|_{\infty}^{p}\right)\right)^{1 / p} .
$$

We prove existence and uniqueness of solutions to Eq.(4.3) in $\mathcal{B}_{p, K, T}$ :

Lemma 10.1. Let $T>0, p>1$, and $M>1$. For sufficiently large $K$, there exists a unique solution $u_{t} \in \mathcal{B}_{p, K, T}$ to Eq.(4.3) with initial data $u_{0}$.

Proof. We define the map $\mathcal{F}: \mathcal{B}_{p, K, T} \rightarrow \mathcal{B}_{p, K, T}$ by (see Eq.4.3)

$$
\mathcal{F}(X)_{t}=e^{t \mathcal{L}} u_{0}+\int_{0}^{t} e^{(t-s) \mathcal{L}} P_{M}\left(\left|X_{s}\right|\right)\left|X_{s}\right|^{2 q} X_{s} \mathrm{~d} s+\int_{0}^{t} e^{(t-s) \mathcal{L}} \xi_{s} \mathrm{~d} w_{s} .
$$

If $\mathbf{E}\left(\left\|u_{0}\right\|_{\infty}^{p}\right)<\infty$ then obviously $\mathcal{F}$ maps $\mathcal{B}_{p, K, T}$ into itself. We define

$$
\begin{aligned}
& \mathcal{N}\left(|x|^{2}\right)=-(b+\mathrm{i} \beta) P_{M}(|x|)|x|^{2 q} \\
& \mathcal{G}_{1}(x, y)=\frac{1}{2}\left(\mathcal{N}\left(|x|^{2}\right)+\mathcal{N}\left(|y|^{2}\right)+\left(|x|^{2}+|y|^{2}\right) \int_{0}^{1} \mathcal{N}^{\prime}\left(t|x|^{2}+(1-t)|y|^{2}\right) \mathrm{d} t\right) \\
& \mathcal{G}_{2}(x, y)=x y \int_{0}^{1} \mathcal{N}^{\prime}\left(t|x|^{2}+(1-t)|y|^{2}\right) \mathrm{d} t .
\end{aligned}
$$

If $X$ and $Y$ are arbitrary elements of $\mathcal{B}_{p, K, T}$, then

$$
\begin{aligned}
& \|\mathcal{F}(X)-\mathcal{F}(Y)\|^{p} \\
& =\sup _{0 \leq t \leq T} \mathbf{E}\left(\left\|\int_{0}^{t} e^{(t-s)(\mathcal{L}-K)}\left(\mathcal{G}_{1}\left(X_{s}, Y_{s}\right) e^{-K s}\left(X_{s}-Y_{s}\right)+\mathcal{G}_{2}\left(X_{s}, Y_{s}\right) e^{-K s}\left(\overline{X_{s}}-\overline{Y_{s}}\right)\right) \mathrm{d} s\right\|_{\infty}^{p}\right) \\
& \leq C M^{2 q p}(K-c)^{-p}\|X-Y\|^{p} .
\end{aligned}
$$

The map $\mathcal{F}$ is thus a contraction on $\mathcal{B}_{p, K, T}$ if $K>C^{\prime} M^{2 q}$. This proves the existence and the uniqueness of a solution $u_{t}$ to Eq.(4.3).

To be able to treat solutions to Eq.(4.5) as solutions to Eq.(4.2) for some time, we use the following bounds on the stopping times $\tau(R)$ defined by Eq.4.4): 
Lemma 10.2. There is a $C>0$ such that the following holds almost surely for all $R>1$ :

$$
\tau(R) \geq C R^{-2 q} \log R
$$

Proof. This follows immediately from Lemma 10.1 since we can take any $M>R$.

We next show that solutions of Eq.(2.1) are also uniquely defined on $\mathrm{L}_{\delta, y}^{2}$, using that bounded functions form a dense subset.

Lemma 10.3. The semi-flow $\Phi_{\omega}^{t}$ extends almost surely to a bounded continuous semi-flow on $\mathrm{L}_{\delta, y}^{2}$ for any $\delta>0$ and $y \in \mathbf{R}^{d}$.

Proof. We apply the non-propagation estimate of Ginibre and Velo [GV1]. Let $u_{0}$ and $v_{0}$ be two functions in $\mathrm{L}_{\delta, y}^{2}$ and denote the corresponding solutions to Eq.(2.1) by $u_{t}$ and $v_{t}$. Their difference $u_{t}-v_{t}$ satisfies (almost surely) the following inequality:

$$
\begin{aligned}
\frac{1}{2} \partial_{t}\left\|\sqrt{\varphi_{\delta, y}}\left(u_{t}-v_{t}\right)\right\|_{2}^{2} \leq & \left(1+\frac{1}{2} \sqrt{1+\alpha^{2}}\right)\left\|\sqrt{\varphi_{\delta, y}}\left(u_{t}-v_{t}\right)\right\|_{2}^{2} \\
& -\operatorname{Re}(1+\mathrm{i} \beta) \int \varphi_{\delta, y}\left(\bar{u}_{t}-\bar{v}_{t}\right)\left(\left|u_{t}\right|^{2 q} u_{t}-\left|v_{t}\right|^{2 q} v_{t}\right) .
\end{aligned}
$$

By [GV]] (Proposition 3.1), Hypothesis 2.1] implies that the last term above is negative. We thus get an estimate of the form $\left\|u_{t}-v_{t}\right\|_{\mathrm{L}_{\delta, y}^{2}} \leq \exp (c t)\left\|u_{0}-v_{0}\right\|_{\mathrm{L}_{\delta, y}^{2}}$

This and Lemma 4.3 prove that $\Phi_{\omega}^{t}$ is uniformly bounded and continuous on $\mathrm{L}_{\delta, y}^{2}$ for any $\delta>0$ and $y \in \mathbf{R}^{d}$ if we define $u_{t}=\lim _{n \rightarrow \infty} u_{t}^{(n)}$ where $u_{0}^{(n)}$ is a Cauchy sequence of bounded functions approaching $u_{0}$.

\section{Compact Embedding for Local Spaces}

In this section, we give a proof of Relation (2.7) which is a trivial adaptation of [Ad], Theorem 6.53, p.174. More precisely we prove the embedding (2.7) to be Hilbert-Schmidt. Let $\left\{e_{n}\right\}_{n \in \mathbf{N}}$ be a complete orthonormal basis of $\mathrm{H}_{\delta, y}^{m+k}$. Let $\left\{Q_{n}\right\}_{n \in \mathbf{N}}$ be a countable cover of $\mathbf{R}^{d}$ by balls of radius 1 . Let $x \in Q_{n}$, let $\alpha \leq m$ and define the bounded linear operator $D_{x}^{\alpha}$ on $\mathrm{H}_{\delta, y}^{m+k}$ by

$$
D_{x}^{\alpha}(u)=\nabla^{\alpha} u(x)
$$

Its norm is (by Sobolev embedding) bounded by

$$
\left\|D_{x}^{\alpha}(u)\right\|_{\mathrm{H}_{\delta, y}^{m+k}}^{2} \leq \max _{0 \leq \alpha \leq m} \sup _{x \in Q_{n}}\left|\nabla^{\alpha} u(x)\right|^{2} \leq \frac{C}{\inf _{x \in Q_{n}} \varphi_{\delta, y}(x)}\|u\|_{\mathrm{H}_{\delta, y}^{m+k}}^{2} .
$$

By Riesz' Lemma, $D_{x}^{\alpha}(\cdot)=\left(v_{x}^{\alpha}, \cdot\right)_{\mathrm{H}_{\delta, y}^{m+k}}$ for some vector $v_{x}^{\alpha}$ and

$$
\sum_{n=1}^{\infty}\left|\nabla^{\alpha} e_{n}(x)\right|^{2}=\sum_{n=1}^{\infty}\left|\left(e_{n}, v_{x}^{\alpha}\right)_{\mathrm{H}_{\delta, y}^{m+k}}\right|^{2}=\left\|v_{x}^{\alpha}\right\|_{\mathrm{H}_{\delta, y}^{m+k}}^{2}
$$


Thus the Hilbert-Schmidt norm of the embedding map is

$$
\sum_{n=1}^{\infty}\left\|e_{n}\right\|_{\mathrm{H}_{\delta^{\prime}, y}^{m}}^{2}=\sum_{\alpha \leq m} \int_{\mathbf{R}^{d}}\left\|v_{x}^{\alpha}\right\|_{\mathrm{H}_{\delta^{\prime}, y}^{m+k}}^{2} \varphi_{\delta^{\prime}, y}(x) \mathrm{d} x \leq m \sum_{n=1}^{\infty} \int_{Q_{n}} \frac{C \varphi_{\delta^{\prime}, y}(x)}{\inf _{z \in Q_{n}} \varphi_{\delta, y}(z)} \mathrm{d} x,
$$

which is finite whenever $\delta^{\prime}>\delta$.

\section{Proof of Lemma 4.6}

The proof can be found in [BGO, Mi] and is summarised below. We decompose the plane into countably many sets $Q(m, n)$ of unit area and use the bounds $\varphi_{\delta, y}(x) \leq \exp (-\delta|x-y|) \leq$ $e \varphi_{\delta, y}(x)$. For simplicity we assume $\delta=1$ and we drop it from our notation (if Lemma 4.6 is true for $\delta=1$ then it is true for all $\delta>0$ by scaling, possibly with different constants). We simply write $\int_{D} f$ for $\int_{D} f(x) \mathrm{d} x$ for $D \subset \mathbf{R}^{2}$. We have

$$
\int_{\mathbf{R}^{2}} \varphi_{y}\left|\Delta\left(|f|^{2 q} f\right) \overline{\Delta f}\right| \leq C \sum_{m, n} e^{-|n|} \int_{Q(m, n)}|\Delta f||f|^{2 q-1}\left(|f||\Delta f|+|\nabla f|^{2}\right),
$$

where $\bigcup_{m} Q(m, n) \supset\left\{x \in \mathbf{R}^{2}: n-\frac{1}{2} \leq|x-y| \leq n+\frac{1}{2}\right\}$. We estimate each summand using Hölder and Gagliardo-Nirenberg inequalities. For any $p, r$ with $p^{-1}+r^{-1}=1$ and in particular for $r=1+1 / q$ and $p=1+q$, we get:

$$
\begin{aligned}
& \int_{Q(m, n)}|\Delta f \| f|^{2 q-1}\left(|f||\Delta f|+|\nabla f|^{2}\right) \\
& \leq c_{1}\|\Delta f\|_{2 p}\left(\|f\|_{2 p q /(p-1)}^{2 q}\|\Delta f\|_{2 p}+\|f\|_{2 p q /(p-1)}^{2 q-1}\|\nabla f\|_{4 p q /(p+q-1)}^{2}\right) \\
& \leq c_{2}\|\Delta f\|_{2 p}\left(\|f\|_{2 p q /(p-1)}^{2 q}\|\Delta f\|_{2 p}+\|f\|_{2 p q /(p-1)}^{2 q-1}\left(\|f\|_{2 p q /(p-1)}^{1 / 2}\|\Delta f\|_{2 p}^{1 / 2}\right)^{2}\right) \\
& =c_{3}\|\Delta f\|_{2 p}^{2}\|f\|_{2 q r}^{2 q} \\
& \leq c_{4}\left\|\nabla^{3} f\right\|_{2}^{2(2 q+2) /(2 q+3)}\|f\|_{2(q+1)}^{2(q+1 /(2 q+3))} \\
& \leq K^{-1}\left\|\nabla^{3} f\right\|_{2}^{2}+c_{5} K\|f\|_{2(q+1)}^{4 q^{2}+6 q+2} .
\end{aligned}
$$

By summing up all contribution to (12.1) we arrive at

$$
\begin{aligned}
& \int_{\mathbf{R}^{2}} \varphi_{y}\left|\Delta\left(|f|^{2 q} f\right) \overline{\Delta f}\right| \\
& \leq C K^{-1} \sum_{m, n} e^{-|n|} \int_{Q(m, n)}\left|\nabla^{3} f\right|^{2}+C^{\prime} K \sum_{m, n} e^{-|n|}\left(\int_{Q(m, n)}|f|^{2(q+1)}\right)^{\eta} \\
& \leq \widetilde{C} K^{-1} \int_{\mathbf{R}^{2}} \varphi_{y}\left|\nabla^{3} f\right|^{2}+C^{\prime \prime} K \sum_{n} n e^{-|n|}\left(\sup _{y} \int_{\mathbf{R}^{2}} \varphi_{y}|f|^{2(q+1)}\right)^{\eta} \\
& =\widetilde{C} K^{-1} \int_{\mathbf{R}^{2}} \varphi_{y}\left|\nabla^{3} f\right|^{2}+C^{\prime \prime \prime} K\left(\sup _{y} \int_{\mathbf{R}^{2}} \varphi_{y}|f|^{2(q+1)}\right)^{\eta},
\end{aligned}
$$

which proves Lemma 4.6 . 
Stochastic Ginzburg-Landau Equations

\section{References}

[Ad] Adams R.A., Sobolev Spaces, Academic Press, New York, 1975.

[Ar] Arnold L., Random Dynamical Systems, Springer, Berlin Heidelberg, 1998.

[BGO] Bartucelli M.V., Gibbon J.D., Oliver M., Length scales in solutions of the complex GinzburgLandau equation, Physica D 89 (1996), 267-286.

[BKL] Bricmont J., Kupiainen A., Lefèvre R., Ergodicity of the 2D Navier-Stokes Equations with Random Forcing, Preprint (2000).

[C1] Collet P., Thermodynamic limit of the Ginzburg-Landau equations, Nonlinearity 7 (1994), 11751190.

[C2] Collet P., Extended Dynamical Systems, Doc. Math. Extra Volume ICM III (1998), 123-132.

[CDF] Crauel H., Debussche A., Flandoli F., Random Attractors J. Dyn. Diff. Equ. 9 (1997), 307-341.

[CE1] Collet P., Eckmann J.-P., Extensive Properties of the Complex Ginzburg-Landau Equation, Commun. Math. Phys. 200 (1999), 699-722.

[CE2] Collet P., Eckmann J.-P., The definition and measurement of the topological entropy per unit volume in parabolic PDEs Nonlinearity 12 (1999), 451-473.

[CE3] Collet P., Eckmann J.-P., Topological entropy and $\varepsilon$-entropy for damped hyperbolic equations, Preprint (1999).

[DZ1] Da Prato G., Zabczyk J., Stochastic equations in infinite dimensions, Cambridge University Press, Cambridge, 1992.

[DZ2] Da Prato G., Zabczyk J., Ergodicity for infinite-dimensional systems, Cambridge University Press, Cambridge, 1996.

[EH] Eckmann J.-P., Hairer M., Invariant Measures for Stochastic PDE's on Unbounded Domains, Preprint (2000).

[F1] Funaki T., The Reversible measures of Multi-Dimensional Ginzburg-Landau Type Continuum Model, Osaka J. Math. 28 (1991), 463-494.

[F2] Funaki T., Regularity Properties for Stochastic Partial Differential Equations of Parabolic Type, Osaka J. Math. 28 (1991), 495-516.

[FM] Flandoli F., Maslowski, B. Ergodicity of the 2-D Navier-Stokes equation under random perturbations, Commun. Math. Phys. 172 (1995), 119-141.

[GV1] Ginibre J., Velo G., The Cauchy Problem in Local Spaces for the Complex Ginzburg-Landau Equation I. Compactness Methods, Physica D 95 (1996), 191-228.

[GV2] Ginibre J., Velo G., The Cauchy Problem in Local Spaces for the Complex Ginzburg-Landau Equation II. Contraction Methods, Commun. Math. Phys. 187 (1997), 45-79.

[KH] Katok A., Hasselblatt B., Introduction to the Modern Theory of Dynamical Systems, Cambridge University Press, Cambridge, 1995.

[Kr] Krylov N.V., An analytic approach to SPDEs, in Stochastic partial differential equations: six perspectives, R.A. Carmona and B. Rozovskii eds., Amer. Math. Soc., Providence, RI, 1999.

[KS] Kuksin S.B., Shirikyan A., Stochastic Dissipative PDE's and Gibbs Measures, Preprint (2000).

[KT] Kolmogorov A.N., Tikhomirov V.M., $\varepsilon$-entropy and $\varepsilon$-capacity of sets in functional spaces, in Selected Works of A.N. Kolmogorov, Vol III, A.N. Shiryayev, ed., Kluwer, Dordrecht, 1993.

[Ku] Kuksin S.B., Stochastic Nonlinear Schrödinger Equation. 1. A priori Estimates, Proc. Steklov Inst. Math. 225 (1999), 219-242.

[LO] Levermore C.D., Oliver M., The complex Ginzburg-Landau equation as a model problem, in Dynamical systems and probabilistic methods in partial differential equations, P. Deift et al., 
eds., Amer. Math. Soc., Providence, RI, 1996.

[LQ] Liu P.-D., Qian M., Smooth Ergodic Theory of Random Dynamical Systems, Lecture Notes in Mathematics, 1606, Springer, Berlin Heidelberg, 1995.

[Ma] Mattingly J.C., Ergodicity of 2D Navier-Stokes equations with random noise and large viscosity, Commun. Math Phys. 206 (1999) 273-288.

[Mi] Mielke A., Bounds for the solutions of the complex Ginzburg-Landau equation in terms of the dispersion parameters, Physica D 117 (1998) 106-116.

[Ro] Rougemont J., $\varepsilon$-Entropy Estimates for Driven Parabolic Equations, Preprint (2000).

[Ru] Ruelle D., Large Volume Limit of the Distribution of Characteristic Exponents in Turbulence, Commun. Math. Phys. 87 (1982) 287-302.

[S] Sinai Ya.G., Two Results Concerning Asymptotic Behaviour of Solutions of the Burgers Equation, J. Statist. Phys. 64 (1991), 1-12.

[VF] Vishik M.J., Fursikov A.V., Mathematical Problems of Statistical Hydromechanics, Kluwer, Dordrecht, 1988.

[Y1] Yosida K., Functional Analysis, Sixth edition, Springer, Berlin New York, 1980.

[Y2] Young L.-S., Ergodic Theory of Chaotic Dynamical Systems, in XIIIth International Congress of Mathematical Physics (ICMP'97), Brisbane, Internat. Press, Cambridge, MA, 1999. 